\title{
Josephson physics of spin-orbit-coupled elongated Bose-Einstein condensates
}

\author{
M. A. Garcia-March, ${ }^{1}$ G. Mazzarella, ${ }^{2}$ L. Dell'Anna, ${ }^{2}$ B. Juliá-Díaz, ${ }^{1,3}$ L. Salasnich, ${ }^{2}$ and A. Polls ${ }^{1}$ \\ ${ }^{1}$ Departament d'Estructura i Constituents de la Materia, Universitat de Barcelona, Diagonal 645, 08028 Barcelona, Spain \\ ${ }^{2}$ Dipartimento di Fisica e Astronomia "Galileo Galilei” and CNISM, Università di Padova, Via Marzolo 8, 35131 Padova, Italy \\ ${ }^{3}$ ICFO-Institut de Ciències Fotòniques, Parc Mediterrani de la Tecnologia, 08860 Barcelona, Spain
}

(Received 3 February 2014; published 6 June 2014)

\begin{abstract}
We consider an ultracold bosonic binary mixture confined in a quasi-one-dimensional double-well trap. The two bosonic components are assumed to be two hyperfine internal states of the same atom. We suppose that these two components are spin-orbit coupled to each other. We employ the two-mode approximation starting from two coupled Gross-Pitaevskii equations and derive a system of ordinary differential equations governing the temporal evolution of the interwell population imbalance of each component and of the polarization, which is the imbalance of the total populations of the two species. From this set of equations we disentangle the different macroscopic quantum tunneling and self-trapping scenarios occurring for both population imbalances and the polarization in terms of the interplay between the interatomic interactions and the other relevant energies in the problem, like the spin-orbit coupling or the conventional tunneling term. We find a rich dynamics in all three variables and discuss the experimental feasibility of such a system.
\end{abstract}

DOI: 10.1103/PhysRevA.89.063607

PACS number(s): 03.75.Lm, 03.75.Mn, 67.85.-d

\section{INTRODUCTION}

In the last few years artificial spin-orbit (SO) coupling has been realized in the laboratory with both neutral bosonic systems [1] and fermionic atomic gases [2,3]. These achievements have stimulated theoretical efforts to understand the role of the SO coupling with Rashba [4] and Dresselhaus [5] terms in the physics of ultracold atoms.

Spin-orbit-coupled Bose-Einstein condensates (BECs) have been considered in Refs. [6-10], where the authors have determined the zero-temperature phase diagram and studied the excitation spectrum in uniform systems. Bosons in two SO-coupled hyperfine states have been investigated in different contexts, for instance by exploring different confinements and geometries, e.g., two-dimensional (2D) periodic geometries [11-13], a tight 2D harmonic potential plus a generic one-dimensional (1D) loose potential [14], and confinements in quasi-1D parabolic traps [15]. A large variety of vortex structures, Skyrmions, and other unconventional BECs has been discussed in this context [16,17]. Also the richer strongly correlated quantum Hall phases stemming from the spin-orbit coupling (which can be regarded as a non-Abelian external field) have been recently discussed $[18,19]$. On the fermionic side, the experimental realization of the SO coupling has produced a growing interest in the study of its role in the crossover from the Bardeen-Cooper-Schrieffer state of weakly bound Fermi pairs to the BEC of molecular dimers both for a 3D uniform Fermi gas [20-22] and in the 2D case [21-23].

One of the richest scenarios opens up when two internal hyperfine states of the same bosonic atom are coupled to each other by means of two counterpropagating laser beams and confined by a one-dimensional double well. This framework represents the ideal arena to analyze the atomic counterpart of the Josephson effect which occurs in superconductoroxide-superconductor junctions [24] with bosonic binary mixtures [25-32]. It is worth noting that Josephson physics with bosonic mixtures seems within reach for a number of experimental groups which have in recent years studied single-component Josephson physics [33-40].
The aim of the present work is to study the Josephson oscillations with SO-coupled Bose-Einstein condensates. In this case, the boson dynamics is ruled by two coupled 3D Gross-Pitaevskii equations, the couplings consisting in the intra- and interspecies interactions and spin-orbit coupling. By employing the two-spatial-mode approximation [41,42], neglecting the interatomic interactions between bosons in different wells, and assuming that the Rashba and the Dresselhaus velocities are equal, one derives a system of ordinary differential equations (ODEs). The solution of these ODEs provides the temporal evolution of the relevant variables of the problem. These are the two fractional population imbalances $z_{k}$, defined as the difference in the occupation of the two wells for each bosonic component labeled by $k=1,2$. On top of these we define the total population imbalance $z_{12}$ between the bosons in species 1 and those in species 2 . We term this latter variable the polarization, i.e., zero polarization implies an equal amount of atoms populating the components 1 and 2, while the system is fully polarized if all atoms populate either one of the two species. The dynamical evolution of $z_{12}$ gives the interchange of atoms between the two bosonic components and is therefore directly related to the SO coupling.

We numerically solve the aforementioned ODEs and study the temporal behavior of the three $z$ 's and their corresponding canonically conjugated phases. We focus on the interplay between the boson-boson interaction and the interwell spinorbit coupling (the intrawell SO coupling is zero in first approximation) in determining the importance of the interchange of atoms between the two bosonic species on the two canonical effects: self-trapping and macroscopic tunneling phenomena. In particular, we point out that an analog of the macroscopic quantum self-trapping that one observes for the variable $z_{k}[27,30]$ exists for the polarization $z_{12}$ as well. During the $z_{12}$ self-trapped dynamics, on the average, one bosonic component is more populated than the other one.

There are thus two major aspects to be explored in this problem. First, the interplay of spin-orbit coupling and 
tunneling phenomena, which has for instance been recently discussed at the single-particle level for an electron in a double quantum dot [43], and secondly, the role played by the spin-orbit coupling in the presence of atom-atom interactions. The former has been explored in detail in a recent work by Zhang and co-workers [44]. There, they concentrated on the effect of the spin-orbit coupling in the Josephson regime, describing a spin-Josephson current induced by the spin-orbit coupling. The effect of interactions was explored only in some limiting cases. In the present article, our focus will be set on the effect of atom-atom interactions, exploring all the scenarios in due detail. In particular we provide here a thorough analysis of the macroscopic quantum self-trapping (MQST) which will be shown to take place in both in the spin polarization and the population imbalances of each species.

The article is organized in the following way. First, in Sec. II we describe the single-particle Hamiltonian of the system, following the experimental realizations of Refs. [1,33], for the implementation of the spin-orbit interaction and doublewell potential, respectively. In Sec. III we present the meanfield description of the problem. In Sec. IV the two-mode approximation is exploited to derive the ordinary differential equations that we use to describe the dynamics of our system. In Sec. $\mathrm{V}$ we study the effect of the spin-orbit term on the macroscopic quantum tunneling and self-trapping. A summary and conclusions are provided in Sec. VI.

\section{DOUBLE-WELL PHYSICS WITH ARTIFICIAL SPIN-ORBIT COUPLING}

The physics of ultracold atoms confined in a double-well potential with spin-orbit coupling can be studied with current state-of-the-art techniques. We will consider for the spin-orbit coupling a similar setup to that in the experiment [1]. The external double-well potential can in principle be implemented simultaneously, as in Ref. [33].

To engineer the spin-orbit coupling we follow the experimental scheme of Ref. [1]. Thus, we consider a pair of Raman lasers-propagating along $\hat{x}+\hat{y}$ (with $\hat{x}$ and $\hat{y}$ the unitary vectors of the $x$ and $y$ axes) and $-\hat{x}+\hat{y}$, respectively, so that the beams intersect at an angle equal to $90^{\circ}$ - which couple two internal hyperfine states of the same bosonic atom, for instance the $m_{F}=0,1$ components of an ${ }^{87} \mathrm{Rb}$ $F=1$ spinor condensate. These two states, $|\uparrow\rangle_{b}=\left|m_{F}=0\right\rangle$ and $|\downarrow\rangle_{b}=\left|m_{F}=1\right\rangle$ ( $b$ stands for bare), differ in energy by the Zeeman shift $\hbar \omega_{Z}$. The two lasers [with frequency difference $\Delta \omega_{L}=\left(\omega_{Z}+\delta\right)$ and wave number $\left.k_{L}\right]$ are detuned by a frequency $\delta$ from the Raman resonance and couple the states $|\uparrow\rangle_{b}$ and $|\downarrow\rangle_{b}$ with the Raman strength $\hbar \Omega_{R}$. Then the single-particle (SP) quantum Hamiltonian can be written as [1]

$$
\begin{aligned}
H_{\mathrm{SP}}= & {\left[\frac{\mathbf{p}^{2}}{2 m}+U(\mathbf{r})\right] \sigma_{0}+v_{R}\left(p_{x} \sigma_{y}-p_{y} \sigma_{x}\right) } \\
& +v_{D}\left(p_{x} \sigma_{y}+p_{y} \sigma_{x}\right)+\frac{\hbar \Omega_{R}}{2} \sigma_{z}+\frac{\hbar \delta}{2} \sigma_{y},
\end{aligned}
$$

where $\mathbf{p}=\left(p_{x}, p_{y}, p_{z}\right)=-i \hbar\left(\partial_{x}, \partial_{y}, \partial_{z}\right)$ is the linear momentum operator, $U(\mathbf{r})$ is the external trapping potential, $v_{R}$ and $v_{D}$ are, respectively, the Rashba and Dresselhaus velocities, $\sigma_{0}$ is the $2 \times 2$ identity matrix, and $\sigma_{x}, \sigma_{y}$, and $\sigma_{z}$ are the
Pauli matrices. In the recent experiments [1-3] one has $v_{R}=v_{D} \equiv v$, which is the configuration that we shall consider in the present paper.

We observe that the Hamiltonian (1) can be obtained from that written in the bare pseudospin basis $|\uparrow\rangle_{b}$ and $|\downarrow\rangle_{b}$ by applying to this, first, the transformation $U=$ $\left[\left[\begin{array}{ll}e^{-i k_{L} x} & 0],\left[0 e^{i k_{L} x}\right.\end{array}\right]\right.$ that corresponds to introduce the dressed pseudospin basis $|\uparrow\rangle=e^{-i k_{L} x}|\uparrow\rangle_{b}$ and $|\downarrow\rangle=e^{i k_{L} x}|\downarrow\rangle_{b}$. The Hamiltonian so achieved is the same (apart from the external trapping term) appearing in Eq. (2) of [44]. By performing on this Hamiltonian the global pseudospin rotation $\sigma_{z} \rightarrow \sigma_{y}$, $\sigma_{y} \rightarrow \sigma_{x}$, and $\sigma_{x} \rightarrow \sigma_{z}$, one obtains the Hamiltonian (1). The relation between our variables and the ones used in [44] is provided in Appendix A. It is also worth noticing that the use of the dressed bases is what makes the Raman coupling, proportional to $\Omega_{R}$, look like a detuning term, and vice versa.

As in Ref. [33], the double-well potential could be implemented with a dipole trap complemented with two optical lasers. The standing wave produces a double-well potential with a separation of the wells of the order of $5 \mu \mathrm{m}$. The details are provided in Ref. [45].

\section{MEAN-FIELD DESCRIPTION: GROSS-PITAEVSKII EQUATIONS}

We consider a dilute binary condensate with a spin-orbit coupling term as described above, whose single-particle Hamiltonian is given by Eq. (1). Due to the assumption $v_{R}=$ $v_{D} \equiv v$ (see the previous section), the spin-orbit coupling is effective just in one dimension, i.e., the $x$ direction. Then the ultracold atomic cloud is further assumed to be confined in the transverse $(y, z)$ plane by a strong harmonic potential with frequency $\omega_{\perp}$ and in the axial ( $x$ ) direction by a generic weak potential $V(x)$, namely,

$$
U(\mathbf{r})=V(x)+\frac{1}{2} m \omega_{\perp}^{2}\left(y^{2}+z^{2}\right) .
$$

The choice of such a trapping potential allows us to restrict our discussions to $1 \mathrm{D}$ and focus on the dynamics of atoms along the $x$ axis by considering practically frozen the dynamics in the $(y, z)$ plane.

Under the hypothesis that the boson-boson interactions (intra- and interspecies) can be described by a contact potential, the two time-dependent 3D Gross-Pitaevskii equations (GPEs) which control the spatial-temporal evolution of the binary condensate are given by

$$
\begin{aligned}
i \partial_{t} \psi_{k}= & {\left[-\frac{1}{2} \nabla^{2}+V(x)+\frac{1}{2}\left(y^{2}+z^{2}\right) \pm \Gamma+2 \pi \tilde{g}_{k}\left|\psi_{k}\right|^{2}\right.} \\
& \left.+2 \pi \tilde{g}_{12}\left|\psi_{3-k}\right|^{2}\right] \psi_{k} \mp\left(\gamma \partial_{x}+i \tilde{\delta}\right) \psi_{3-k}
\end{aligned}
$$

where $k=1,2$. The sign in front of $\Gamma$ is plus (minus) when $k=1(k=2)$, while the second sign ambiguity is solved with minus (plus) for $k=1(k=2)$. In Eq. (3) lengths, times, and energies are written in units of $a_{\perp}=\sqrt{\hbar /\left(m \omega_{\perp}\right)}, \omega_{\perp}^{-1}$, and $\hbar \omega_{\perp}$, respectively. Here $\psi_{k}(x, y, z, t)$ is the macroscopic wave function of the $k$ th atomic hyperfine state $(k=1,2)$. The number of atoms populating each hyperfine state can be computed at any time as

$$
\iiint\left|\psi_{k}(x, y, z, t)\right|^{2} d \mathbf{r}=N_{k}(t) .
$$


The strengths of the intra- and interspecies interactions are given by

$$
\tilde{g}_{k} \equiv 2 a_{k} / a_{\perp}, \quad \tilde{g}_{12} \equiv 2 a_{12} / a_{\perp},
$$

where $a_{k}$ and $a_{12}$ are the $s$-wave scattering lengths pertaining, respectively, to the intraspecies and interspecies interactions. Note that $\gamma=2 v /\left(a_{\perp} \omega_{\perp}\right)$ is the dimensionless SO coupling, and $\Gamma=\Omega_{R} /\left(2 \omega_{\perp}\right)$ and $\tilde{\delta}=\delta /\left(2 \omega_{\perp}\right)$ are the dimensionless Raman and detuning frequencies, respectively.

These GPEs can be derived with the ordinary variational procedure from the Lagrangian density,

$$
L=L_{0}+L_{\mathrm{SO}}+L_{I}
$$

where

$$
L_{0}=\sum_{k=1,2} \psi_{k}^{*}\left[i \partial_{t}+\frac{1}{2} \nabla^{2}-V(x)-\frac{1}{2}\left(y^{2}+z^{2}\right)\right] \psi_{k}
$$

is the Lagrangian density of the noninteracting binary condensate,

$$
\begin{aligned}
L_{\mathrm{SO}}= & -\Gamma\left(\left|\psi_{1}\right|^{2}-\left|\psi_{2}\right|^{2}\right)+\gamma\left(\psi_{1}^{*} \partial_{x} \psi_{2}-\psi_{2}^{*} \partial_{x} \psi_{1}\right) \\
& +i \tilde{\delta}\left(\psi_{1}^{*} \psi_{2}-\psi_{2}^{*} \psi_{1}\right)
\end{aligned}
$$

is the SO-coupling contribution, and

$$
L_{I}=-\pi \tilde{g}_{1}\left|\psi_{1}\right|^{4}-\pi \tilde{g}_{2}\left|\psi_{2}\right|^{4}-2 \pi \tilde{g}_{12}\left|\psi_{1}\right|^{2}\left|\psi_{2}\right|^{2}
$$

is the term due to $s$-wave interactions between the bosonic atoms.

By assuming a cloud strongly localized in the transverse plane and weakly localized in the axial direction [see the above discussion about the trapping potential (2)], we can derive a system of effective one-dimensional Gross-Pitaevskii equations by adopting the usual Gaussian ansatz for the wave functions $\psi_{k}(k=1,2)$ in the directions $y$ and $z$, that is,

$$
\psi_{k}(x, y, z, t)=\frac{1}{\sqrt{\pi} a_{\perp}} \exp \left\{-\frac{y^{2}+z^{2}}{2 a_{\perp}^{2}}\right\} f_{k}(x, t),
$$

where the complex functions $f_{k}(x, t)(k=1,2)$ are dynamical fields, obeying the normalization $\int_{-\infty}^{+\infty}\left|f_{k}(x, t)\right|^{2} d x=N_{k}$, as follows from Eqs. (4) and (10).

Inserting the ansatz (10) in the Lagrangian density $L$ given by Eq. (6) [its contributions being the right-hand sides of Eqs. (7)-(9)] and performing the integration in the transverse directions ( $y$ and $z$ ) starting from $\int d \mathbf{r} L$ we obtain the effective 1D Lagrangian

$$
\begin{aligned}
\bar{L}= & \int d x\left\{\left[\sum_{k=1,2} f_{k}^{*}\left(i \partial_{t}+\frac{1}{2} \partial_{x}^{2}\right) f_{k}-[1+V(x)]\left|f_{k}\right|^{2}\right.\right. \\
& \left.-\frac{\tilde{g}_{k}}{2}\left|f_{k}\right|^{4}\right]-\tilde{g}_{12}\left|f_{1}\right|^{2}\left|f_{2}\right|^{2}-\Gamma\left(\left|f_{1}\right|^{2}-\left|f_{2}\right|^{2}\right) \\
& \left.+\gamma\left(f_{1}^{*} \partial_{x} f_{2}-f_{2}^{*} \partial_{x} f_{1}\right)+i \tilde{\delta}\left(f_{1}^{*} f_{2}-f_{2}^{*} f_{1}\right)\right\}
\end{aligned}
$$

By varying $\bar{L}$ with respect to $f_{k}^{*}$ we get the following system formed by two coupled 1D GPEs embedded in the following equation:

$$
\begin{aligned}
i \partial_{t} f_{k}= & {\left[-\frac{1}{2} \partial_{x}^{2}+V(x) \pm \Gamma+\tilde{g}_{k}\left|f_{k}\right|^{2}+\tilde{g}_{12}\left|f_{3-k}\right|^{2}\right] f_{k} } \\
& \mp\left(\gamma \partial_{x}+i \tilde{\delta}\right) f_{3-k}
\end{aligned}
$$

where $k=1,2$, and the ambiguities about the signs are solved as was done for Eq. (3).

\section{BIMODAL APPROXIMATION}

Let us suppose that the potential $V(x)$ on the right-hand side of Eq. (2) is a symmetric double-well potential $V_{\mathrm{DW}}(x)$. To describe the dynamics by a finite-mode approximation, we use a two-mode ansatz for each wave function $f_{k}$ (recall $k=1,2$ ), as originally introduced in [42]:

$$
f_{k}(x, t)=\Psi_{k}^{L}(t) \phi_{k}^{L}(x)+\Psi_{k}^{R}(t) \phi_{k}^{R}(x) .
$$

The functions $\phi_{k}^{\alpha}(x)(\alpha=L, R)$ which we consider as real functions as was done for example in [29-31], are singleparticle wave functions tightly localized in the $\alpha$ th well, while the time dependence is encoded in $\Psi_{k}^{\alpha}(t) \equiv \sqrt{N_{k}^{\alpha}(t)} e^{i \theta_{k}^{\alpha}(t)}$, where the total number of particles in the $k$ th species is given by $N_{k}^{L}(t)+N_{k}^{R}(t)=\left|\Psi_{k}^{L}(t)\right|^{2}+\left|\Psi_{k}^{R}(t)\right|^{2} \equiv N_{k}(t)$. The functions $\phi_{k}^{\alpha}(x)$ satisfy the following orthonormalization conditions: $\int d x\left[\phi_{k}^{\alpha}(x)\right]^{2}=1$ and $\int d x \phi_{k}^{L}(x) \phi_{k}^{R}(x)=0$.

At this point, we exploit the two-mode approximation for $f_{k}(x, t)$ given by Eq. (13) in each of the two coupled one-dimensional Gross-Pitaevskii equations (12). By leftmultiplying both the left-hand and the right-hand sides of the $f_{k}$ 1D GPE for $\phi_{k}^{\alpha}(x)$ and integrating in the $x$ direction, one obtains the equations of motion for $N_{k}^{\alpha}$ and $\theta_{k}^{\alpha}$ (note that here one has to take into account the above orthonormalization conditions). We retain only the integrals with wave functions localized in the same well for the boson-boson interactions and terms related to the Raman and detuning terms. Conversely, due to the derivative coupling, both the SO coupling in the same and different wells has to be considered. The equations of motion for $N_{k}^{\alpha}$ and $\theta_{k}^{\alpha}$ thus read

$$
\begin{aligned}
\dot{N}_{k}^{\alpha}= & -2 J_{k} \sqrt{N_{k}^{\alpha^{\prime}} N_{k}^{\alpha}} \sin \left(\theta_{k}^{\alpha^{\prime}}-\theta_{k}^{\alpha}\right) \\
& +2 S_{12}^{\alpha} \sqrt{N_{3-k}^{\alpha} N_{k}^{\alpha}} \sin \left(\theta_{k}^{\alpha}-\theta_{3-k}^{\alpha}\right) \\
& \pm 2 \bar{S}_{12}^{\alpha, \alpha^{\prime}} \sqrt{N_{3-k}^{\alpha^{\prime}} N_{k}^{\alpha}} \sin \left(\theta_{k}^{\alpha}-\theta_{3-k}^{\alpha^{\prime}}\right) \\
& 2 D_{12}^{\alpha} \sqrt{N_{3-k}^{\alpha} N_{k}^{\alpha}} \cos \left(\theta_{k}^{\alpha}-\theta_{3-k}^{\alpha}\right), \\
\dot{\theta}_{k}^{\alpha}= & -\left(U_{k} N_{k}^{\alpha}+\epsilon_{k}+U_{12} N_{3-k}^{\alpha} \pm \Gamma\right) \\
& +J_{k} \sqrt{\frac{N_{k}^{\alpha^{\prime}}}{N_{k}^{\alpha}} \cos \left(\theta_{k}^{\alpha}-\theta_{k}^{\alpha^{\prime}}\right)} \\
& +S_{12}^{\alpha} \sqrt{\frac{N_{3-k}^{\alpha}}{N_{k}^{\alpha}}} \cos \left(\theta_{k}^{\alpha}-\theta_{3-k}^{\alpha}\right) \\
& \pm \bar{S}_{12}^{\alpha, \alpha^{\prime}} \sqrt{\frac{N_{3-k}^{\alpha^{\prime}}}{N_{k}^{\alpha}}} \cos \left(\theta_{k}^{\alpha}-\theta_{3-k}^{\alpha^{\prime}}\right) \\
& \pm D_{12}^{\alpha} \sqrt{\frac{N_{3-k}^{\alpha}}{N_{k}^{\alpha}}} \sin \left(\theta_{k}^{\alpha}-\theta_{3-k}^{\alpha}\right) .
\end{aligned}
$$

The sign in front of the $D_{12}$ term in Eq. (14) is minus (plus) for $k=1$ (2). In Eq. (15) the sign in front of $\Gamma$ is plus (minus) 
for $k=1$ (2). In both equations the \pm sign in front of $\bar{S}_{12}^{\alpha, \alpha^{\prime}}$ is plus (minus) for the component 1 (2).

The following constants are introduced in terms of the single-particle functions $\phi_{k}^{\alpha}$ :

$$
\begin{aligned}
\epsilon_{k}^{\alpha} & =\int d x\left[\phi_{k}^{\alpha}(x)\left(-\frac{1}{2} \partial_{x}^{2}+V_{\mathrm{DW}}(x)\right) \phi_{k}^{\alpha}(x)\right]+1, \\
U_{k} & =\tilde{g}_{k} \int d x\left(\phi_{k}^{\alpha}\right)^{4}, \\
J_{k} & =-\int d x\left[\phi_{k}^{L}(x)\left(-\frac{1}{2} \partial_{x}^{2}+V_{\mathrm{DW}}(x)\right) \phi_{k}^{R}(x)\right], \\
U_{12} & =\tilde{g}_{12} \int d x\left(\phi_{1}^{\alpha}\right)^{2}\left(\phi_{2}^{\alpha}\right)^{2}, \\
S_{12}^{\alpha} & =\gamma \int d x \phi_{1}^{\alpha}(x) \partial_{x} \phi_{2}^{\alpha}(x), \\
\bar{S}_{12}^{\alpha, \alpha^{\prime}} & =\gamma \int d x \phi_{1}^{\alpha}(x) \partial_{x} \phi_{2}^{\alpha^{\prime}}(x), \\
D_{12}^{\alpha} & =\tilde{\delta} \int d x \phi_{1}^{\alpha}(x) \phi_{2}^{\alpha}(x) .
\end{aligned}
$$

$\epsilon_{k}^{\alpha}$ is the kinetic plus the potential energy (due to the external trapping) of the bosonic component $k(k=1,2)$ inside well $\alpha(\alpha=L, R) . U_{k}$ and $J_{k}$ are the intrawell interaction and the tunneling amplitude between the two wells for the $k$ species, respectively. $U_{12}$ is the interspecies interaction between bosons in the same well. The two terms which genuinely have the spin-orbit-coupling term in the single-particle Hamiltonian are $S_{12}^{\alpha}$ and $\bar{S}_{12}^{\alpha, \alpha^{\prime}}$, which are for bosons within the same well and different wells, respectively. Finally, $D_{12}^{\alpha}$ is the intrawell coupling between different components due to the detuning $\delta$.

In obtaining the equations of motion (14) and (15), we have used the double-well left-right symmetry so that $\epsilon_{k}^{L}=\epsilon_{k}^{R} \equiv \epsilon_{k}, U_{k}^{L}=U_{k}^{R} \equiv U_{k}$, and $U_{12}^{L}=U_{12}^{R} \equiv U_{12}$. We have employed, moreover, the following properties: By integrating by parts we have both $S_{12}^{\alpha}=-S_{21}^{\alpha}$ and $\bar{S}_{12}^{\alpha, \alpha^{\prime}}=$ $-\bar{S}_{21}^{\alpha^{\prime}, \alpha}$, and finally that $\bar{S}_{12}^{\alpha, \alpha^{\prime}}=-\bar{S}_{12}^{\alpha^{\prime}, \alpha}$. We are assuming that $m_{1}=m_{2}=m$; thus it is possible to work with the same localized modes for both components, that is, $\phi_{1}^{\alpha}=\phi_{2}^{\alpha}=\phi^{\alpha}$ $(\alpha=L, R)$. If this is the case, $\epsilon_{1}=\epsilon_{2}=\epsilon, J_{1}=J_{2}=J$, and $D_{12}=\tilde{\delta}$. This also implies that $S_{12}^{\alpha}$ and $S_{21}^{\alpha}$ vanish. After defining $S_{+}=\bar{S}_{12}^{L, R}=-\bar{S}_{21}^{R, L}$ and $S_{-}=\bar{S}_{12}^{R, L}=-\bar{S}_{21}^{L, R}$, we can rewrite the equations of motion (14) and (15) as

$$
\begin{gathered}
\dot{N}_{k}^{\alpha}=-2 J \sqrt{N_{k}^{\alpha^{\prime}} N_{k}^{\alpha}} \sin \left(\theta_{k}^{\alpha^{\prime}}-\theta_{k}^{\alpha}\right) \\
+2 S_{ \pm(\mp)} \sqrt{N_{3-k}^{R(L)} N_{k}^{L(R)}} \sin \left(\theta_{k}^{L(R)}-\theta_{3-k}^{R(L)}\right) \\
\mp 2 \tilde{\delta} \sqrt{N_{3-k}^{\alpha} N_{k}^{\alpha}} \cos \left(\theta_{k}^{\alpha}-\theta_{3-k}^{\alpha}\right) \\
\dot{\theta}_{k}^{\alpha}=-\left(U_{k} N_{k}^{\alpha}+U_{12} N_{3-k}^{\alpha} \pm \Gamma\right)+J \sqrt{\frac{N_{k}^{\alpha^{\prime}}}{N_{k}^{\alpha}}} \cos \left(\theta_{k}^{\alpha}-\theta_{k}^{\alpha^{\prime}}\right) \\
+S_{ \pm(\mp)} \sqrt{\frac{N_{3-k}^{R(L)}}{N_{k}^{L(R)}} \cos \left(\theta_{k}^{L(R)}-\theta_{3-k}^{R(L)}\right)} \\
\pm \tilde{\delta} \sqrt{\frac{N_{3-k}^{\alpha}}{N_{k}^{\alpha}}} \sin \left(\theta_{k}^{\alpha}-\theta_{3-k}^{\alpha}\right) .
\end{gathered}
$$

The upper (lower) sign in these equations holds for $k=1$ (2). For the SO-coupling term $\left(S_{ \pm}\right)$, the symbol without (with) parentheses holds for the equation of motion of the occupation $N_{k}^{\alpha}$ or $\theta_{k}^{\alpha}$ with $\alpha=L(R)$. In Appendix A we show how to derive these equations as the semiclassical limit of the exact bimodal many-body Hamiltonian. The equations (17) and (18) rule the temporal evolution of $N_{k}^{\alpha}$ and $\theta_{k}^{\alpha}$. From these equations, it can be seen that the hopping amplitude $J$, the spin-orbit-coupling strength $S$, and the detuning $\tilde{\delta}$ are responsible for coherent phenomena related to the interwell transfer of the same bosonic species, coupling of different components in different wells, and coupling of different components in the same well, respectively. Nevertheless, the role played by these three parameters will be analyzed in more detail in the following sections, where we discuss the dynamics by analyzing a wide class of scenarios.

As detailed in Appendix B, a set of population imbalances and corresponding phase differences provide an appropriate set of variables to study the interplay between tunneling and spinorbit coupling. These variables allow one to make the connection with the usual Josephson dynamics in binary mixtures and thus to better understand the role played by the spin-orbit coupling. We define one population imbalance between wells for each component, $z_{1}=\left(N_{1}^{L}-N_{1}^{R}\right) / N, z_{2}=\left(N_{2}^{L}-N_{2}^{R}\right) / N$, and a global population imbalance between components, which can be seen as polarization, $z_{12}=\left(N_{1}-N_{2}\right) / N$, with $N_{k}=N_{k}^{L}+N_{k}^{R}$. The corresponding phases are $\theta_{1}=\theta_{1}^{R}-\theta_{1}^{L}$, $\theta_{2}=\theta_{2}^{R}-\theta_{2}^{L}$, and $\theta_{12}=\theta_{2}^{L}+\theta_{2}^{R}-\left(\theta_{1}^{L}+\theta_{1}^{R}\right)$.

\section{JOSEPHSON DYNAMICS IN THE SPIN-ORBIT-COUPLED DOUBLE WELL}

For the numerical results discussed in this section, we consider $N=10^{5}{ }^{87} \mathrm{Rb}$ atoms, and that the wavelength of the two counterpropagating lasers is $\lambda \approx 10^{3} \mathrm{~nm}$. Following Ref. [1], we introduce natural units for the momentum and energy as $k_{L}=\sqrt{2} \pi / \lambda$ and $E_{L}=\hbar^{2} k_{L}^{2} /(2 m)$. The SO coupling is given by $v=E_{L} / \hbar k_{L}$ and therefore for $\omega_{\perp}=400 \pi \mathrm{Hz}$ one obtains $\gamma=3.37$. Notice that $S_{ \pm}$is defined as an overlap integral [see Eq. (16)]. To keep the model simple we approximate the four on-site modes by Gaussian wave functions. In this case, the overlap integral is proportional to $\exp \left(-d^{2}\right), d$ being the distance between the minimum of each well and the origin. Then both $S_{ \pm}$and $J$ can be tuned by varying the distance between the wells.

The SO coupling $v$ is independent of the detuning $\delta$ and the Raman coupling $\Omega_{R}$. We assume that $\Omega_{R}$ can be tuned in the interval $\left[0,7 E_{L} / \hbar\right]$, and then $\Gamma_{\max }=19.92 . \bar{\delta}$ is proportional to $\exp \left(-d^{2}\right)$ and can thus also be tuned by varying $d$. In the following, we take the scattering lengths $a_{1} \simeq a_{2} \simeq 101.8 a_{0}$ [46], with $a_{0}$ the Bohr atom radius which gives $U_{\text {ref }} \approx 0.0012$, where $U_{\text {ref }}=\left(2 a_{00} / a_{\perp}\right) \int d x\left[\phi^{\alpha}(x)\right]^{4}$, $a_{00}=101.8 a_{0}$, and the integral is performed on the whole real axis. We refer all variables in the rest of the paper to this value of the interactions. We define $\Lambda_{J} \equiv U_{\text {ref }} N / J$, which is the usual variable quantifying the ratio between atom-atom interaction and tunneling in a single-component bosonic Josephson junction. Similarly, we define the quantities $\Lambda_{S} \equiv U_{\text {ref }} N / S_{+}$, and $\Lambda_{D} \equiv U_{\text {ref }} N / \tilde{\delta}$. Finally, we assume that 
the interactions can be tuned with respect to the reference, and therefore we define $C_{U} \equiv U / U_{\text {ref }}$ and $C_{U_{12}} \equiv U_{12} / U_{\text {ref }}$.

Note that in this part of the paper, we shall take $U_{12}=0$ to make the effect of the spin-orbit coupling as clear and neat as possible. Let us note, however, that the effect of the repulsion between species has been discussed thoroughly in Refs. [25$32,47]$, where it was found that the conventional Josephson dynamics is crucially modified by this term, even leading to measure synchronization in some limits [48]. In particular, the interspecies interaction induces the presence of additional fixed points in the problem which are related to the repulsion between components. Nevertheless, we take into account the effect of the boson-boson repulsion in Sec. VD.

\section{A. Some considerations about fixed points}

Inspection of Hamiltonian (B1) [or its many-body counterpart, Eqs. (A5), (A6), and (A8)] permits one to identify three different processes which interchange atoms between wells or components. The first one is the usual tunneling between the two wells and is given by the term $J \sqrt{N_{k}^{\alpha} N_{k}^{\alpha^{\prime}}} \cos \left(\theta_{k}^{\alpha}-\theta_{k}^{\alpha^{\prime}}\right)$ in Eq. (B1). The second one is associated with the SO terms proportional to $S_{ \pm}$in Eq. (B1) and couples the atoms of one species located in one well to the atoms of the second species located in the other well. The third one is given by the terms corresponding to the detuning $\tilde{\delta}$ in Eq. (B1). This is a coupling between atoms of different species in the same well. Finally, we note that the Raman frequency $\Gamma$ introduces an energy gap between the two species in Eq. (B1). In this work, we study the effect of the SO coupling, detuning, and Raman frequencies in the well-known Josephson dynamics in double wells. We focus on the case in which the most populated species has a certain population imbalance and study the effect of the dynamics of this species on the second initially balanced species. To understand this problem, let us discuss briefly the fixed points of Eqs. (B9)-(B12) when SO coupling and detuning frequencies are considered.

In the absence of interactions and when all terms other than the tunneling energy vanish, the fixed points are the usual ones at $\left(z_{k}^{0}, \theta_{k}^{0}\right)=(0, n \pi), n \in \mathbb{Z}$. In such a case, there is no process that can produce interchange of atoms between the two components. Therefore $z_{12}$ remains constant at its initial value. This picture changes when the other two processes are considered. In the presence of the SO coupling and tunneling, when all other terms vanish, one can prove that Eqs. (B9)(B12) vanish for $\left(z_{k}^{0}, \theta_{k}^{0}\right)=\left(z_{12}^{0}, \theta_{12}^{0}\right)=(0,0)$. When initially $z_{12}$ is different from zero and all other variables vanish, the population imbalances $z_{k}$ and the phase $\theta_{12}$ will remain at their initial values, while the equations of motion can be reduced to

$$
\begin{gathered}
\dot{z}_{12}=-2 S_{+} \sin \bar{\theta} \sqrt{1-z_{12}^{2}}, \\
\dot{\bar{\theta}}=2 S_{+} \cos \bar{\theta} \frac{z_{12}}{\sqrt{1-z_{12}^{2}}},
\end{gathered}
$$

with $\bar{\theta}=\left(\theta_{1}+\theta_{2}\right) / 2$. Therefore, both $z_{12}$ and $\bar{\theta}$ will oscillate during the evolution. In such a case, we observe numerically that the growth of $\theta_{1}$ and $\theta_{2}$ is unbounded, with opposite signs. In addition, when initially all variables are zero except for $z_{k}$, the polarization $z_{12}$ remains at its initial value at zero. On the other hand, $\left(\theta_{1}+\theta_{2}\right) / 2$ and $z_{1}+z_{2}$ will oscillate, with the latter bounded by $\pm\left[z_{1}(0)+z_{2}(0)\right]$, while $\theta_{12}$ also oscillates. We observe numerically that $z_{1}-z_{2}=z_{1}(0)-z_{2}(0)$ throughout the evolution.

In the presence of only detuning, Eqs. (B9)-(B12) vanish for $\left(z_{k}^{0}, \theta_{k}^{0}\right)=(0,0)$, but now it is necessary that $\left(z_{12}^{0}, \theta_{12}^{0}\right)=$ $(0, \pi)$. When initially $z_{12}$ is different from zero, $\theta_{12}=\pi$, and all other variables vanish, the population imbalances $z_{k}$ and the phase $\theta_{12}$ will remain at their initial values, while the equations of motion can be reduced to

$$
\begin{aligned}
& \dot{z}_{12}=-2 \tilde{\delta} \cos \tilde{\theta} \sqrt{1-z_{12}^{2}}, \\
& \dot{\tilde{\theta}}=-4 \tilde{\delta} \sin \tilde{\theta} \frac{z_{12}}{\sqrt{1-z_{12}^{2}}},
\end{aligned}
$$

with $\tilde{\theta}=\left(\theta_{1}-\theta_{2}\right) / 2$. Therefore, both $z_{12}$ and $\tilde{\theta}$ will oscillate during the evolution. If $z_{k}$ are different from zero initially, while $z_{12}$ is zero, both $\left(\theta_{1}-\theta_{2}\right) / 2$ and $z_{1}-z_{2}$ will oscillate, with the latter bounded by $\pm\left[z_{1}(0)-z_{2}(0)\right]$. Now, $z_{12}$ remains at its initial value, $\theta_{12}$ also oscillates, and we observe numerically that $z_{1}+z_{2}=z_{1}(0)+z_{2}(0)$.

In the next section we study how the fixed-point analysis briefly discussed above can help to understand the dynamics when the most populated species has a certain population imbalance, while the second species is balanced, that is, initially $z_{12}$ and $z_{1}$ are nonzero, while $z_{2}$ is zero. To illustrate this situation we consider in all numerical examples to be discussed in the next sections that initially $z_{1}=0.518$, $z_{2}=0.002, z_{12}=0.64$, and all initial phases are zero unless explicitly indicated.

\section{B. Macroscopic quantum tunneling and self-trapping in the presence of spin-orbit coupling}

We first assume $\bar{\delta}=\Gamma=0$, and study the effect of the SO coupling $v$. This coupling is associated with the kinetic moment $p_{x}$ or $p_{y}$ of the atoms in each species [see Eq. (1)]. As we have shown, when the single-particle $3 \mathrm{D}$ potential can be reduced effectively to a $1 \mathrm{D}$ double well, where the dynamics in transverse directions is essentially frozen, the SO coupling, proportional to $S_{ \pm}$, becomes apparent in a nontrivial way in the equations of motion.

The SO-coupling term allows for the complete transfer of the atoms of species 1 in the left well to species 2 in the right well [see Figs. 1(a) and 1(b)], when no other term is considered. In the presence of a tunneling term which dominates the SO coupling [see Figs. 1(c) and 1(d)], $z_{1}$ shows fast Rabi oscillations, where its corresponding phase is bounded. This can be understood in view of Eqs. (B9) and (B11), as in the presence of a tunneling which dominates over $S_{ \pm}$, these equations will vanish only if $\theta_{k}=0, \pi, \ldots$ Therefore, in this case the growth of $\theta_{k}$ cannot be unbounded and has to oscillate around $\theta_{k}=0$. A small transfer of atoms between the two components still occurs, but it is not enough to transfer all population from component 1 to component 2 before it tunnels to the other well. If $S_{ \pm}$are comparable to $J$, the two effects are combined, the transfer of atoms between components 

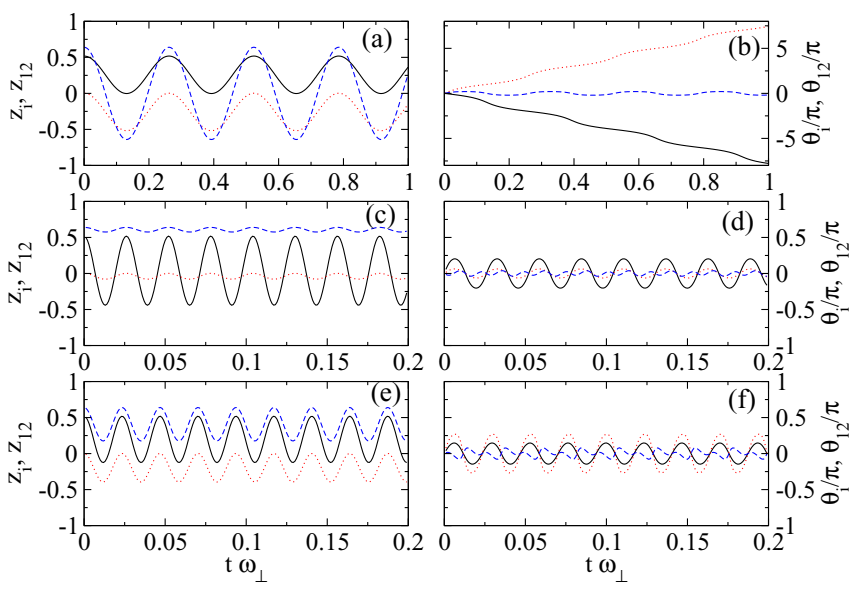

FIG. 1. (Color online) Macroscopic quantum tunneling in the presence of a SO term. (a) Population imbalances $z_{1}$ (solid black line) and $z_{2}$ (dotted red line), and polarization $z_{12}$ (dashed blue line) when $\Lambda_{S}=10$ in the absence of tunneling $(J=0)$ ). (b) Corresponding phases. (c), (d) Population imbalances, polarization, and phases when $\Lambda_{S}=10$ in the presence of tunneling $\left(\Lambda_{J}=1\right)$. (e), (f) The same when $\Lambda_{S}=2$. In all cases, $U=U_{12}=\tilde{\delta}=\Gamma=0$. Initial conditions: $z_{1}(0)=0.518, z_{2}(0)=0.002, z_{12}=0, \theta_{k}(0)=0$ $(k=1,2)$, and $\theta_{12}(0)=0$.

is enlarged, and the tunneling of the atoms of component 1 is reduced, as shown in Figs. 1(e) and 1(f).

The studied tunneling between components, triggered by the spin-orbit-coupling term, is essentially the spin current described in Ref. [44]. An interesting feature of our approach is that in the dressed basis considered in this work, the spin flip is favored whenever the atoms tunnel to the other side; thus, in our approach the effect gets enhanced when starting with most of the atoms in one component and on one side, as occurred in Fig. 1(a).

Let us now illustrate the effect of the interactions on the dynamics induced by the SO coupling shown in Figs. 1(a) and 1(b) corresponding to the absence of hopping and interactions. In the presence of a small interaction term, the transfer of atoms between components associated with the dynamical evolution of the polarization $z_{12}$ still occurs. The interactions only modulate this dynamics only slightly, as shown in Figs. 2(a) and 2(b). Conversely, for larger $U$, self-trapping occurs in all variables, and correspondingly all phases are running [see Figs. 2(c) and 2(d)]. When both tunneling and interactions are different from zero, self-trapping can occur on the polarization $z_{12}$ or the population imbalances $z_{k}$ independently. In Figs. 3(a) and 3(b) we show the dynamics when the interactions dominate over the SO term, but not over the tunneling, which induces self-trapping on $z_{12}$, while $z_{k}$ still oscillates. Increasing the interactions further produces self-trapping also in $z_{k}$, as plotted in Figs. 3(c) and 3(d). We conclude this section with a remark about Figs. 1(a) and 1(b). The phase $\left(\theta_{1}+\theta_{2}\right) / 2$ oscillates accordingly with $\theta_{1}$ and the growth of $\theta_{2}$ is unbounded with opposed sign [see Eq. (19)]. Moreover, because $z_{k}$ are different from zero initially, $z_{1}+z_{2}$ oscillates, while $z_{1}-z_{2}=z_{1}(0)-z_{2}(0)=0.516$ throughout the whole evolution, and $\theta_{12}$ oscillates, in accordance with the discussion in Sec. V A.
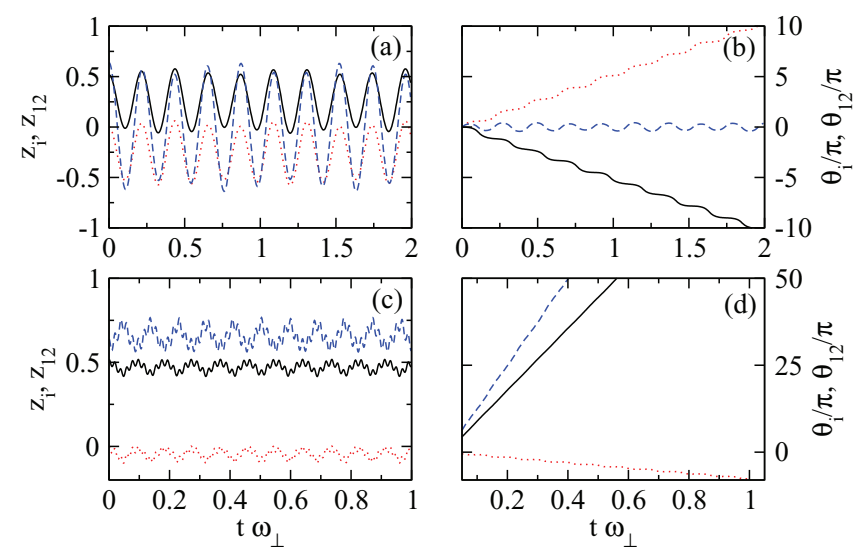

FIG. 2. (Color online) Macroscopic quantum self-trapping in the presence of a SO term. (a) Population imbalances $z_{1}$ and $z_{2}$, and polarization $z_{12}$ when $\Lambda_{S}=10$ when the interactions are small, $C_{U}=$ 1/5. Same color conventions as in Fig. 1. (b) Corresponding phases. (c), (d) Self-trapping induced by the interactions $\left(\Lambda_{S}=10, C_{U}=5\right)$. In all cases, $U_{12}=J=\tilde{\delta}=\Gamma=0$. Initial conditions as in Fig. 1.

\section{Macroscopic quantum tunneling and self-trapping \\ in the presence of the Raman and detuning frequencies}

Let us now discuss the effect of the Raman and detuning frequencies, $\Gamma$ and $\bar{\delta}$, respectively. The detuning frequency induces a local transfer of population between both components. To illustrate this, we represent in Figs. 4(a) and 4(b) the dynamics when all other terms are zero and the tunneling $J$ is very small, when initially $\theta_{12}=\pi$, and all other phases vanish. According to Eq. (21), the corresponding dynamics will be oscillatory around the fixed point, with $\theta_{1}-\theta_{2}$ also oscillating. Because initially $z_{1}=0.518$ and $z_{2}=0.002, z_{1}-z_{2}$ also oscillates with $z_{1}+z_{2}=z_{1}(0)+z_{2}(0)=0.52$ throughout the whole evolution. Again, $\theta_{12}$ also oscillates, in accordance with the discussion in Sec. V A. For larger $J$, the atoms of
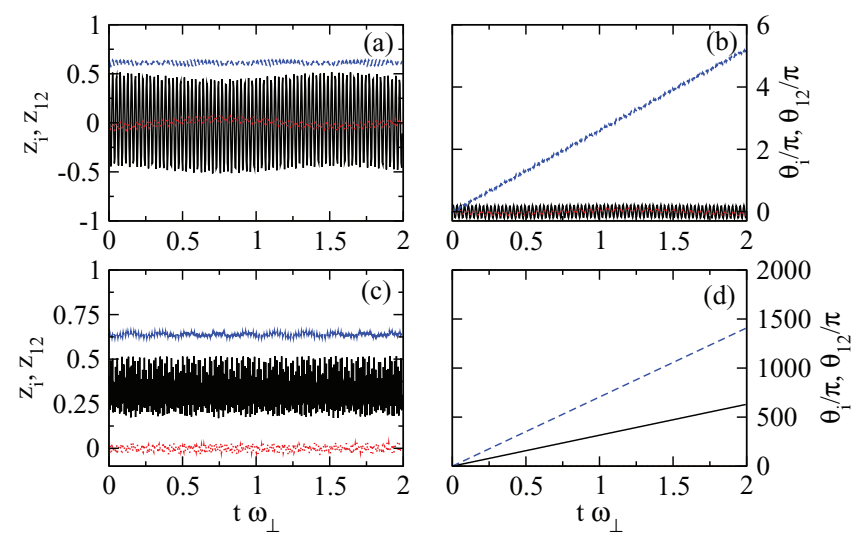

FIG. 3. (Color online) Macroscopic quantum tunneling and selftrapping in the presence of a SO term. (a) Population imbalances $z_{1}$ and $z_{2}$, and polarization $z_{12}$ for $\Lambda_{S}=10$ when the interactions are small $\left(C_{U}=1 / 10\right)$ in the presence of tunneling, $\Lambda_{J}=1$, showing the self-trapping dynamics of $z_{12}$. Same color conventions as in Fig. 1. (b) Corresponding phases. (c), (d) Self-trapping dynamics in all variables when $\Lambda_{S}=10$ and $C_{U}=25$. In all cases, $U_{12}=\tilde{\delta}=\Gamma=0$. Initial conditions as in Fig. 1. 

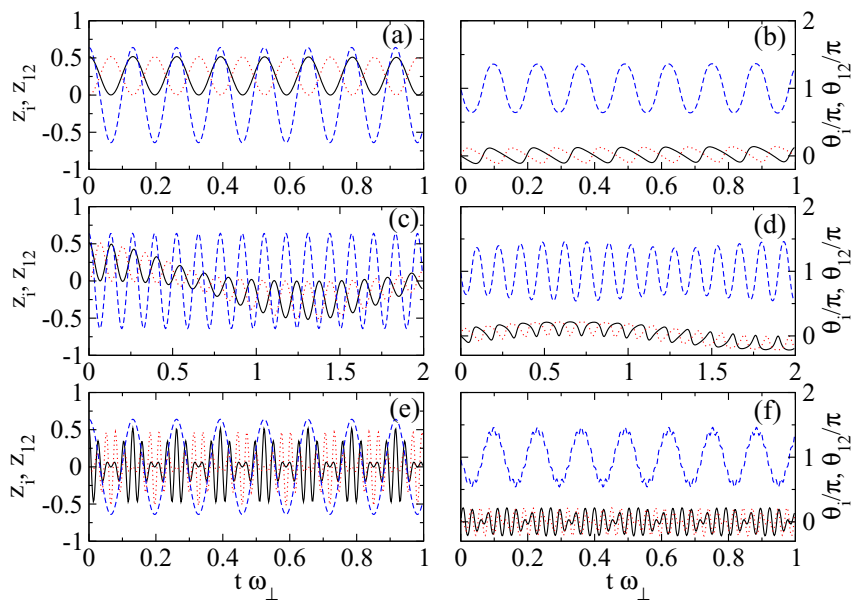

FIG. 4. (Color online) Macroscopic quantum tunneling in the presence of a detuning $\tilde{\delta}$, when the initial phase for the polarization is $\theta_{12}(0)=\pi$. (a) Population imbalances $z_{1}$ and $z_{2}$, and polarization $z_{12}$ for $\Lambda_{D}=5$ when the tunneling term is very small, $\Lambda_{J}=10^{3}$. The same color conventions as in Fig. 1. (b) Corresponding phases. (c), (d) The same when $\Lambda_{J}=10^{2}$, and (e), (f) when $\Lambda_{J}=1$. In all cases, $U=U_{12}=S_{ \pm}=\Gamma=0$. Initial conditions for $z_{k}, \theta_{k}(k=1,2)$, and $z_{12}$ as in Fig. 1.

each species tunnel also to the other well on the same time scales, as shown in Figs. 4(c) and 4(d). Unlike the dynamics in the presence of the SO term, the growth of $\theta_{1}$ and $\theta_{2}$ is not unbounded in the absence of tunneling. Therefore, atoms can still be transformed from component 1 to component 2 in the presence of large tunneling. This effect is quicker if $J$ is increased further, as illustrated in Figs. 4(e) and 4(f). The dynamics of $z_{12}$ is not affected by the Josephson physics. This is due to the fact that the detuning term induces local population transfer, similarly to the transfer of populations among the different Zeeman components in a spinor BEC [49]. In Fig. 5 we reproduce the same cases when initially $\theta_{12}=0$. As this initial condition does not correspond to a fixed point, the phase oscillates abruptly and $z_{12}$ oscillates in the interval $[-1,1]$, as it possibly corresponds to an initial condition close to a separatrix. In Figs. 6(a) and 6(b) we show that the interactions can induce self-trapping in $z_{12}$ when they dominate over the detuning term. If increased further, Figs. 6(c) and 6(d), the interactions induce self-trapping in all variables.

The effect of the Raman frequency on the dynamics associated with $S_{ \pm}$and $\delta$ can be understood in view of the equations of motion for $\theta_{12}$, Eq. (B12), as $\Gamma$ appears as a constant in the equation. Therefore, it introduces an energy gap between the two components, which, when it dominates over the rest of the terms, forces $\theta_{12}$ to be a running phase, similarly to the problem of bosons in an excited level in double wells [50]. In Figs. 7(a) and 7(b) we show the effect of $\Gamma$ on the oscillations of the polarization $z_{12}$ due to the effect of the SO coupling $S_{ \pm}$. We observe that the oscillation of the polarization is reduced with respect to the case of Figs. 1(a) and $1(b)$, and $\theta_{12}$ is a running phase. Then the effect of $\Gamma$ is to inhibit the coupling of the two components associated with the SO term. Similarly, in Figs. 7(c) and 7(d) we observe that $\Gamma$ again reduces [with respect to the case of Figs. 5(e) and 5(f)]
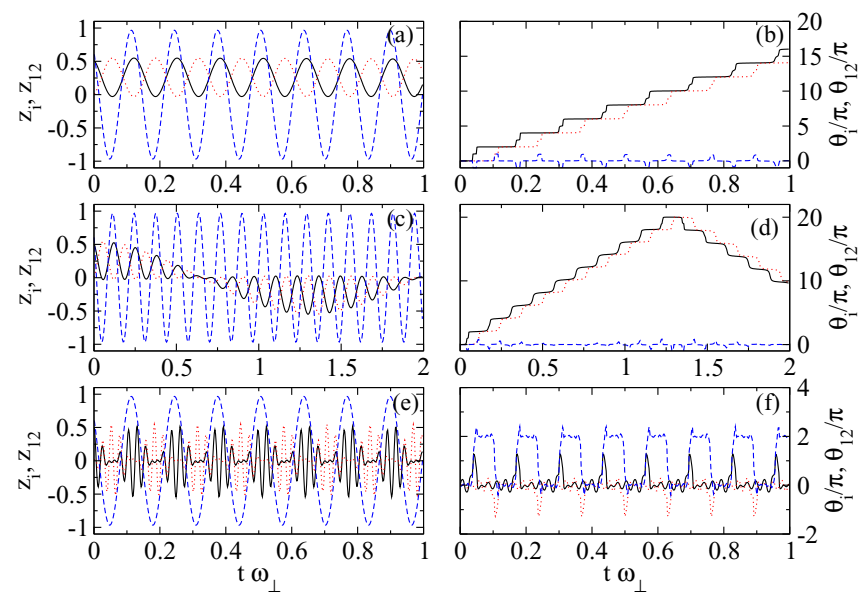

FIG. 5. (Color online) Macroscopic quantum tunneling in the presence of a detuning $\tilde{\delta}$, when the initial phase for the polarization is $\theta_{12}(0)=0$. (a) Population imbalances $z_{1}$ and $z_{2}$, and polarization $z_{12}$ for $\Lambda_{D}=5$ when the tunneling term is very small, $\Lambda_{J}=10^{3}$. The same color conventions as in Fig. 1. (b) Corresponding phases. (c), (d) The same when $\Lambda_{J}=10^{2}$, and (e), (f) when $\Lambda_{J}=1$. In all cases, $U=U_{12}=S_{ \pm}=\Gamma=0$. Initial conditions $z_{k}, \theta_{k}(k=1,2)$, and $z_{12}$ as in Fig. 1.

the oscillations in $z_{12}$ induced by the detuning frequency $\delta$, again decoupling the dynamics of the two components.

\section{Effect of the interspecies interaction}

As noted before, in the previous results we decided to set the interspecies interaction $U_{12}$ to zero, to emphasize the effects of the spin-orbit coupling and Raman and detuning frequencies. In a possible experimental realization along the lines of the recent SO experiments [1] it may be difficult to experimentally achieve this limiting case. For the case of considering two of the Zeeman states of the $F=1{ }^{87} \mathrm{Rb}$ state one has
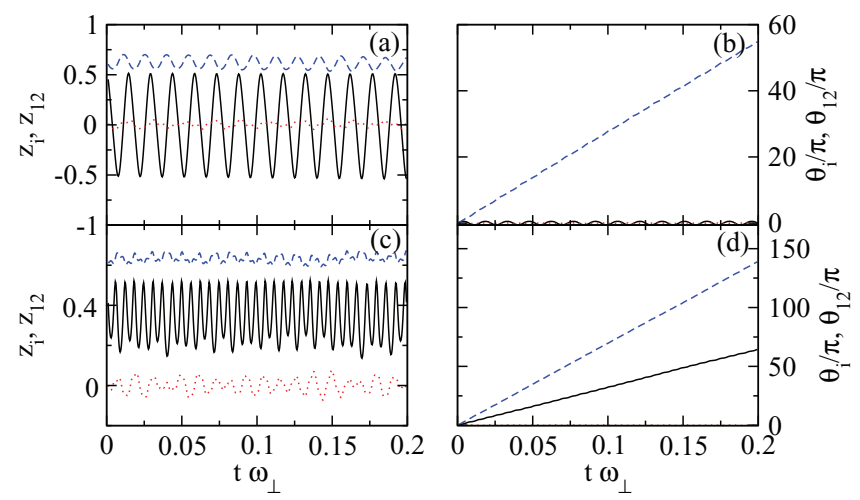

FIG. 6. (Color online) Macroscopic quantum self-trapping in the presence of a detuning $\tilde{\delta}$, when initially $\theta_{12}(0)=0$. (a) Population imbalances $z_{1}$ and $z_{2}$, and polarization $z_{12}$ when $\Lambda_{D}=5, \Lambda_{J}=1$, $C_{U}=10$, and all other terms vanish, showing the self-trapping dynamics of $z_{12}$. The same color conventions as in Fig. 1. (b) Corresponding phases. (c), (d) Self-trapping in all variables when the interactions are increased to $C_{U}=25$. Initial conditions for $z_{k}, \theta_{k}$ $(k=1,2)$, and $z_{12}$ as in Fig. 1 . 

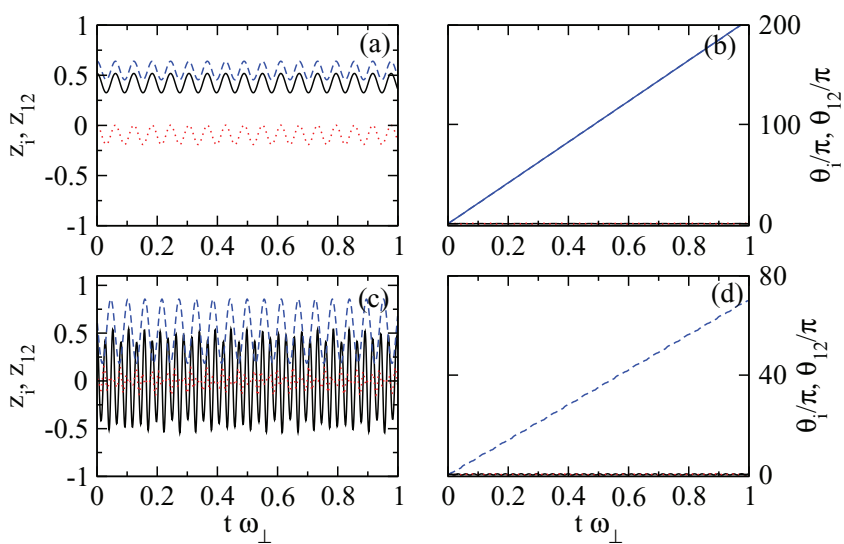

FIG. 7. (Color online) Decoupling induced by the Raman frequency $\Gamma$. (a) Population imbalances $z_{1}$ and $z_{2}$, and polarization $z_{12}$ when $\Lambda_{S}=10, \Gamma=50$, and all other terms vanish. The same color conventions as in Fig. 1. (b) Corresponding phases. (c), (d) The same when $\Lambda_{D}=5, \Gamma=50, \Lambda_{J}=1$, and all other terms vanish. Initial conditions as in Fig. 1.

that $U_{1} \approx U_{2} \approx U_{12}$ [32]. The interspecies interactions have profound and diverse effects on the Josephson dynamics of two components in double wells [25-32,47,50], and therefore an extensive discussion on this topic is out of the scope of this paper. If the interspecies interaction $U_{12}$ is similar to the intraspecies ones $\left(U_{1}\right.$ and $\left.U_{2}\right)$, and for the case considered here of a polarized initial state, the dynamics of the less populated species is crucially influenced by the dynamics of the more populated one [28]. Moreover, for a value of $U_{12}$ above a certain threshold, both species cover the same region in the phase portrait, an effect known as measure
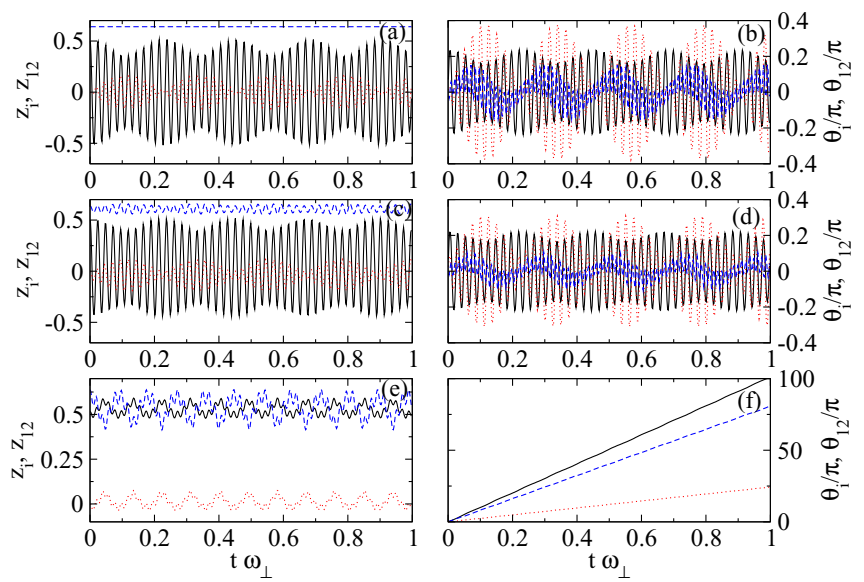

FIG. 8. (Color online) Macroscopic quantum tunneling and selftrapping in the presence of SO coupling and interspecies interactions $U_{12}$. (a) Population imbalances $z_{1}$ and $z_{2}$, and polarization $z_{12}$ when $C_{U}=C_{U_{12}}=1 / 2$ and $\Lambda_{J}=1$, and all other terms vanish, reproducing two-component dynamics in the absence of SO coupling. The same color conventions as in Fig. 1. (b) Corresponding phases. (c), (d) Effect of the SO-coupling term when $\Lambda_{S}=10$. (e), (f) dragging of $z_{2}$ by $z_{1}$ in the self-trapping dynamics, when $\Lambda_{S}=10$, $C_{U}=5, C_{U_{12}}=1$, and all other terms vanish. Initial conditions as in Fig. 1. synchronization (MS) $[47,48]$. In Figs. 8(a) and 8(b) we show the dynamics of the system when all the detunings and the SO-coupling term vanish. Here, the value of $U_{12}$ is below the MS threshold, as can be seen from the fact that $z_{1}$ and $z_{2}$ have different maximum amplitudes. In Figs. 8(c) and 8(d) we have increased the SO term slightly, and some transfer of atoms between the two species occurs. If the interactions dominate over the tunneling and the SO term [for example, as in Figs. 2(c) and 2(d)], all variables are self-trapped and the corresponding phases grow. In Figs. 8(e) and 8(f) we plot the same case as in Figs. 2(c) and 2(d) for nonzero interspecies interactions. Moreover, the evolution of $z_{2}$ is slightly dragged by that of $z_{1}$, an effect which is in accordance with the results of Ref. [28]. We have also observed numerically that the phenomena associated with the detuning and Raman frequencies described above still occur in the presence of interspecies energy.

\section{SUMMARY AND CONCLUSIONS}

The recent developments in ultracold atomic gases, namely, the experimental realization of external bosonic Josephson junctions, together with the artificial creation of spin-orbit coupling for ultracold atoms paved the way to a discussion of the interplay between the two effects in a common setup. As we have described, the conventional macroscopic quantum tunneling or self-trapping scenarios of two bosonic components confined in a double well determine crucially the polarization induced by the spin-orbit coupling. We have shown that the SO coupling transfers atoms between the two components in different wells. This population transfer induced by the SO term depends on how this energy compares with the tunneling and interaction energy. In the macroscopic quantum tunneling limit this transfer is large if it dominates over the tunneling energy. By increasing the interactions we observe that one can induce the self-trapping of the polarization variable and subsequently of the population imbalance. Second, we have studied the effect of the Raman and detuning frequencies. We have shown that the effect of the Raman frequency is to decouple the two components, and thus reduce the population transfer between the two species. On the contrary, the effect of the detuning frequency is a coupling between both components in the same well. Now, this transfer occurs even in the presence of large tunneling energy, although on a different time scale. The interactions produce also self-trapping in all variables if they dominate over the tunneling and detuning. Finally, we have shown that the phenomena associated with the SO coupling and detuning and Raman frequencies are robust in the presence of interspecies interactions.

Note added in proof. Recently, a paper appeared on arXiv analyzing the quantum approach and considering also the weak-interaction limit [51].

\section{ACKNOWLEDGMENTS}

L.D., G.M., and L.S. acknowledge financial support from MIUR (PRIN Grant No. 2010LLKJBX), the University of Padova (Progetto di Ateneo Grant No. CPDA118083) and Cariparo Foundation (Progetto di Eccellenza). G.M. acknowledges financial support also from Progetto Giovani 
of University of Padova. L.D. acknowledges financial support also from MIUR (FIRB Grant No. RBFR12NLNA_002). This work has been supported by Grants No. FIS2011-24154 and No. 2009-SGR1289. B.J.D. is supported by the Ramón y Cajal program.

\section{APPENDIX A: MANY-BODY HAMILTONIAN FOR THE SPIN-ORBIT EFFECT IN DOUBLE WELLS}

The second-quantized Hamiltonian for $N$ interacting twocomponent bosons of equal mass $m$ confined by an external potential $U(\mathbf{r})$ in terms of the creation and annihilation field operators $\hat{\Psi}(\mathbf{r})$ and $\hat{\Psi}^{\dagger}(\mathbf{r})$ in the presence of spin-orbit coupling is

$$
\begin{aligned}
\hat{H}= & \int d \mathbf{r} \hat{\Psi}^{\dagger}(\mathbf{r}) H_{\mathrm{sp}} \hat{\Psi}(\mathbf{r}) \\
& +\frac{1}{2} \int d \mathbf{r} \hat{\Psi}^{\dagger}(\mathbf{r})\left[\int d \mathbf{x}^{\prime} \hat{\Psi}^{\dagger}\left(\mathbf{r}^{\prime}\right) V_{\mathrm{int}}\left(\mathbf{r}-\mathbf{r}^{\prime}\right) \hat{\Psi}(\mathbf{r})\right] \hat{\Psi}(\mathbf{r}),
\end{aligned}
$$

where $V_{\text {int }}\left(\mathbf{r}-\mathbf{r}^{\prime}\right)$ stands for the two-body interaction, and $\hat{\Psi}$ and $\hat{\Psi}^{\dagger}$ are two-component vectors. Here $H_{\text {sp }}$ is

$$
\begin{aligned}
H_{\mathrm{sp}}= & \left(-\frac{\hbar^{2}}{2 m} \nabla^{2}+U(\mathbf{r})\right) \sigma_{0}+v_{R}\left(p_{x} \sigma_{y}-p_{y} \sigma_{x}\right) \\
& +v_{D}\left(p_{x} \sigma_{y}+p_{y} \sigma_{x}\right)+\frac{\Omega_{R}}{2} \sigma_{z}+\frac{\delta}{2} \sigma_{y},
\end{aligned}
$$

with $\sigma_{0}$ the $2 \times 2$ identity matrix, and $\sigma_{x, y, z}$ the Pauli matrices. Let us write $\hat{\Psi}(\mathbf{r})=\left(\hat{\Psi}_{1}(\mathbf{r}), \hat{\Psi}_{2}(\mathbf{r})\right)$, where the index 1 (2) accounts for the first (second) component. We consider contact interactions both for intraspecies and for interspecies interactions. This means that the interatomic potential for the former case is assumed to be $g_{k} \delta\left(\mathbf{r}-\mathbf{r}^{\prime}\right)\left(g_{k}=4 \pi \hbar^{2} a_{k} / m\right.$ with $a_{k}$ the intraspecies $s$-wave scattering length), while for the latter $g_{12} \delta\left(\mathbf{r}-\mathbf{r}^{\prime}\right)\left(g_{12}=4 \pi \hbar^{2} a_{12} / m\right.$ with $a_{12}$ the interspecies $s$-wave scattering length). Then the interacting part of the Hamiltonian can be written in the following way:

$$
\hat{H}_{\mathrm{int}}=\sum_{k=1,2} \frac{g_{k}}{2} \int d \mathbf{r} \hat{\Psi}_{k}^{\dagger} \hat{\Psi}_{k}^{\dagger} \hat{\Psi}_{k} \hat{\Psi}_{k}+g_{12} \int d \mathbf{r} \hat{\Psi}_{1}^{\dagger} \hat{\Psi}_{2}^{\dagger} \hat{\Psi}_{2} \hat{\Psi}_{1} .
$$

We can write the Hamiltonian as $\hat{H}=\hat{H}_{1}+\hat{H}_{2}+\hat{H}_{12}$ with

$$
\hat{H}_{1}=\int d \mathbf{r} \hat{\Psi}_{1}^{\dagger} H_{\mathrm{sp}}^{1} \hat{\Psi}_{1}+\frac{g_{1}}{2} \int d \mathbf{r} \hat{\Psi}_{1}^{\dagger} \hat{\Psi}_{1}^{\dagger} \hat{\Psi}_{1} \hat{\Psi}_{1}
$$

and similarly for species 2. Here, the single-particle Hamiltonian is $H_{\mathrm{sp}}^{k}=\left(-\frac{\hbar^{2}}{2 m} \nabla^{2}+U(\mathbf{r}) \pm \Omega_{R} / 2\right)$ with the plus and minus for 1 and 2 , respectively. The part of the Hamiltonian which couples the two bosonic species is

$$
\begin{aligned}
\hat{H}_{12}= & \hbar \int d \mathbf{r} \hat{\Psi}_{1}^{\dagger}\left[v_{R}\left(-\partial_{x}+i \partial_{y}\right)+v_{D}\left(-\partial_{x}-i \partial_{y}\right)\right] \hat{\Psi}_{2} \\
& +\hbar \int d \mathbf{r} \hat{\Psi}_{2}^{\dagger}\left[v_{R}\left(\partial_{x}+i \partial_{y}\right)+v_{D}\left(\partial_{x}-i \partial_{y}\right)\right] \hat{\Psi}_{1} \\
& +g_{12} \int d \mathbf{r} \hat{\Psi}_{1}^{\dagger} \hat{\Psi}_{2}^{\dagger} \hat{\Psi}_{2} \hat{\Psi}_{1}-i \int d \mathbf{r} \hat{\Psi}_{1}^{\dagger} \frac{\delta}{2} \hat{\Psi}_{2}+i \int d \mathbf{r} \hat{\Psi}_{2}^{\dagger} \frac{\delta}{2} \hat{\Psi}_{1}
\end{aligned}
$$

We assume a separable potential, with harmonic confinement in the $y$ and $z$ directions and a double well in the $x$ direction $U(\mathbf{r})=\frac{1}{2} m \omega_{\perp}^{2}\left(y^{2}+z^{2}\right)+V_{\mathrm{DW}}(x)$. Let us write $\hat{\Psi}_{1}(\mathbf{r})=$ $\hat{a}_{L} \psi_{1}^{L}(\mathbf{r})+\hat{a}_{R} \psi_{1}^{R}(\mathbf{r})$ and $\hat{\Psi}_{2}(\mathbf{r})=\hat{b}_{L} \psi_{2}^{L}(\mathbf{r})+\hat{b}_{R} \psi_{2}^{R}(\mathbf{r})$, with $\hat{a}_{L(R)}$ and $\hat{b}_{L(R)}$ operators annihilating a boson of the species 1 and a boson of the species 2, respectively, in the left (right) well. These single-particle operators obey the usual boson commutation relations. For the orbitals $\psi_{k}^{\alpha}(\mathbf{r})$ we use Gaussian-like functions for the $y$ and $z$ directions (i.e., the ground-state wave function of the harmonic oscillator $m \omega_{\perp}^{2} y^{2} / 2$ times that of the harmonic oscillator $\left.m \omega_{\perp}^{2} z^{2} / 2\right)$ and on-well localized functions $w_{k}^{\alpha}(x)\left(\int d x w_{k}^{\alpha *} w_{k}^{\alpha^{\prime}}=\delta_{\alpha, \alpha^{\prime}}\right)$ in the $x$ direction. In such a way, we have that $\psi_{k}^{\alpha}(\mathbf{r})=\frac{1}{\sqrt{\pi} a_{\perp}} \exp \left(-\frac{y^{2}+z^{2}}{2 a_{\perp}^{2}}\right) w_{k}^{\alpha}(x)$ with $a_{\perp}=\sqrt{\hbar / m \omega_{\perp}}, k=1,2$ and $\alpha=L, R$. Then we obtain

$$
\hat{H}=\hat{H}_{1}+\hat{H}_{2}+\hat{H}_{12} \text {. }
$$

The first two terms are

$$
\hat{H}_{1}=\left(\hat{n}_{1}^{L}+\hat{n}_{1}^{R}\right) \varepsilon_{1}-J_{1} \sum_{\alpha \neq \alpha^{\prime}} \hat{a}_{\alpha}^{\dagger} \hat{a}_{\alpha^{\prime}}+\frac{U_{1}}{2} \sum_{\alpha} \hat{n}_{1}^{\alpha}\left(\hat{n}_{1}^{\alpha}-1\right),
$$

where $\hat{n}_{1}^{\alpha}=\hat{a}_{\alpha}^{\dagger} \hat{a}_{\alpha}$, and similarly for $k=2$. Here and in the following $\sum_{\alpha}$ is a shorthand notation for $\sum_{\alpha=L, R}$. We have used the following constants:

$$
\begin{aligned}
E_{k} & =\int d \mathbf{r} \psi_{k}^{\alpha *}(\mathbf{r})\left(-\frac{\hbar^{2}}{2 m} \nabla^{2}+U(\mathbf{r})\right) \psi_{k}^{\alpha}(\mathbf{r}), \\
\sigma & =\frac{\Omega_{R}}{2} \int d \mathbf{r} \psi_{k}^{\alpha *}(\mathbf{r}) \psi_{k}^{\alpha}(\mathbf{r}), \\
J_{k} & =-\int d \mathbf{r} \psi_{k}^{\alpha *}(\mathbf{r})\left(-\frac{\hbar^{2}}{2 m} \nabla^{2}+U(\mathbf{r})\right) \psi_{k}^{\alpha^{\prime}}(\mathbf{r}), \\
U_{k} & =g_{k} \int d \mathbf{r}\left|\psi_{k}^{\alpha}(\mathbf{r})\right|^{4},
\end{aligned}
$$

and $\varepsilon_{k}=E_{k} \pm \sigma$, where the minus sign holds for $k=2$. The interspecies term is

$$
\begin{aligned}
\hat{H}_{12}= & \sum_{\alpha}\left(S_{12}^{\alpha}+i D_{12}^{\alpha}\right) \hat{a}_{\alpha}^{\dagger} \hat{b}_{\alpha}+\sum_{\alpha \neq \alpha^{\prime}} \bar{S}_{12}^{\alpha, \alpha^{\prime}} \hat{a}_{\alpha}^{\dagger} \hat{b}_{\alpha^{\prime}} \\
& +\sum_{\alpha}\left(S_{21}^{\alpha}+i D_{21}^{\alpha}\right) \hat{b}_{\alpha}^{\dagger} \hat{a}_{\alpha}+\sum_{\alpha \neq \alpha^{\prime}} \bar{S}_{21}^{\alpha, \alpha^{\prime}} \hat{b}_{\alpha}^{\dagger} \hat{a}_{\alpha^{\prime}} \\
& +U_{12} \sum_{\alpha} \hat{n}_{1}^{\alpha} \hat{n}_{2}^{\alpha}
\end{aligned}
$$

with

$$
\begin{aligned}
U_{12} & =g_{12} \int d \mathbf{r} \psi_{1}^{\alpha *}(\mathbf{r}) \psi_{2}^{\alpha *}(\mathbf{r}) \psi_{2}^{\alpha}(\mathbf{r}) \psi_{1}^{\alpha}(\mathbf{r}), \\
S_{12}^{\alpha} & =\int d \mathbf{r} \psi_{1}^{\alpha *} \hbar\left[v_{R}\left(-\partial_{x}+i \partial_{y}\right)+v_{D}\left(-\partial_{x}-i \partial_{y}\right)\right] \psi_{2}^{\alpha}, \\
S_{21}^{\alpha} & =\int d \mathbf{r} \psi_{2}^{\alpha *} \hbar\left[v_{R}\left(\partial_{x}+i \partial_{y}\right)+v_{D}\left(\partial_{x}-i \partial_{y}\right)\right] \psi_{1}^{\alpha}, \\
\bar{S}_{12}^{\alpha, \alpha^{\prime}} & =\int d \mathbf{r} \psi_{k}^{\alpha *} \hbar\left[v_{R}\left(-\partial_{x}+i \partial_{y}\right)+v_{D}\left(-\partial_{x}-i \partial_{y}\right)\right] \psi_{k^{\prime}}^{\alpha^{\prime}},
\end{aligned}
$$




$$
\begin{aligned}
\bar{S}_{21}^{\alpha, \alpha^{\prime}} & =\int d \mathbf{r} \psi_{2}^{\alpha *} \hbar\left[v_{R}\left(\partial_{x}+i \partial_{y}\right)+v_{D}\left(\partial_{x}-i \partial_{y}\right)\right] \psi_{1}^{\alpha^{\prime}}, \\
D_{12}^{\alpha} & =-\int d \mathbf{r} \psi_{1}^{\alpha *} \frac{\delta}{2} \psi_{2}^{\alpha}, \quad D_{21}^{\alpha}=\int d \mathbf{r} \psi_{2}^{\alpha *} \frac{\delta}{2} \psi_{1}^{\alpha} .
\end{aligned}
$$

Nonzero interwell interacting terms have been neglected, as is commonly assumed in standard two- or four-mode Hamiltonians [52-54]. This Hamiltonian conserves the number of atoms as it commutes with the total number operator $\hat{N}=\sum_{k, \alpha} \hat{n}_{k}^{\alpha}$. For $v_{R}=v_{D}=v$ the coefficients given in Eqs. (A9) are the following:

$$
\begin{aligned}
S_{12}^{\alpha} & =-2 \hbar v \int d \mathbf{r} \psi_{1}^{\alpha *}(\mathbf{r}) \partial_{x} \psi_{2}^{\alpha}(\mathbf{r}), \\
S_{21}^{\alpha} & =2 \hbar v \int d \mathbf{r} \psi_{2}^{\alpha *}(\mathbf{r}) \partial_{x} \psi_{1}^{\alpha}(\mathbf{r}) \\
\bar{S}_{12}^{\alpha, \alpha^{\prime}} & =-2 \hbar v \int d \mathbf{r} \psi_{1}^{\alpha *}(\mathbf{r}) \partial_{x} \psi_{2}^{\alpha^{\prime}}(\mathbf{r}) \\
\bar{S}_{21}^{\alpha, \alpha^{\prime}} & =2 \hbar v \int d \mathbf{r} \psi_{2}^{\alpha *}(\mathbf{r}) \partial_{x} \psi_{1}^{\alpha^{\prime}}(\mathbf{r}) \\
D_{12}^{\alpha} & =-\frac{\delta}{2} \int d \mathbf{r} \psi_{1}^{\alpha *}(\mathbf{r}) \psi_{2}^{\alpha}(\mathbf{r}) \\
D_{21}^{\alpha} & =\frac{\delta}{2} \int d \mathbf{r} \psi_{2}^{\alpha *}(\mathbf{r}) \psi_{1}^{\alpha}(\mathbf{r})
\end{aligned}
$$

By integrating by parts, we get that $S_{12}^{\alpha}=S_{21}^{\alpha}$ and $\bar{S}_{12}^{\alpha, \alpha^{\prime}}=\bar{S}_{21}^{\alpha^{\prime}, \alpha}$. We also notice that $\bar{S}_{12}^{L, R}=-\bar{S}_{12}^{R, L}$ and that $D_{12}^{\alpha}=-D_{21}^{\alpha}$. Then, by letting $S=S_{12}^{\alpha}, S_{+}=\bar{S}_{12}^{L, R}=\bar{S}_{21}^{R, L}$, $S_{-}=\bar{S}_{12}^{R, L}=\bar{S}_{21}^{L, R}$, and $\tilde{\delta}=D_{12}^{\alpha}$, we can write Eq. (A8) as

$$
\begin{aligned}
\hat{H}_{12}= & \sum_{\alpha}(S+i \tilde{\delta}) \hat{a}_{\alpha}^{\dagger} \hat{b}_{\alpha}+\text { H.c. }+U_{12} \sum_{\alpha} \hat{n}_{1}^{\alpha} \hat{n}_{2}^{\alpha} \\
& +S_{+} \hat{a}_{L}^{\dagger} \hat{b}_{R}+\text { H.c. }+S_{-} \hat{b}_{L}^{\dagger} \hat{a}_{R}+\text { H.c. }
\end{aligned}
$$

We have checked that this Hamiltonian conserves the number of atoms. Notice that there are four different processes that interchange atoms between both species. The first two, associated with $S$ and $\tilde{\delta}$, interchange atoms between the $k$ and $l$ components located in the same well. The third term, associated with $S_{+}$, interchanges atoms of component $k$ located in the left well and atoms of component $l$ located in the right well. The last term, $S_{-}$, transforms atoms of $k$ in the right well to atoms of $l$ in the left well, and vice versa. From the Heisenberg equations of motion for the operators $\hat{a}_{\alpha}$ and $\hat{b}_{\alpha}$,

$$
i \hbar \frac{d \hat{a}_{\alpha}}{d t}=\left[\hat{a}_{\alpha}, \hat{H}\right] \quad \text { and } \quad i \hbar \frac{d \hat{b}_{\alpha}}{d t}=\left[\hat{b}_{\alpha}, \hat{H}\right]
$$

one can obtain

$$
\begin{aligned}
i \hbar \frac{d \hat{a}_{\alpha}}{d t}= & -J_{1} \hat{a}_{\alpha^{\prime}}+U_{1} \hat{n}_{1}^{\alpha} \hat{a}_{\alpha}+\varepsilon_{1} \hat{a}_{\alpha}+U_{12} \hat{n}_{2}^{\alpha} \hat{a}_{\alpha} \\
& -S \hat{b}_{\alpha}-\bar{S}_{ \pm} \hat{b}_{\alpha^{\prime}}-i \tilde{\delta} \hat{b}_{\alpha}, \\
i \hbar \frac{d \hat{b}_{\alpha}}{d t}= & -J_{2} \hat{b}_{\alpha^{\prime}}+U_{2} \hat{n}_{2}^{\alpha} \hat{b}_{\alpha}+\varepsilon_{2} \hat{b}_{\alpha}+U_{12} \hat{n}_{1}^{\alpha} \hat{b}_{\alpha} \\
& -S \hat{a}_{\alpha}-\bar{S}_{\mp} \hat{a}_{\alpha^{\prime}}+i \tilde{\delta} \hat{a}_{\alpha},
\end{aligned}
$$

and their corresponding Hermitian conjugates. The upper (lower) sign in the $S_{ \pm}$coefficients apply to $\alpha=L(R)$. To reconcile the definition of the coefficients with the one given in Eqs. (16) we have redefined all coefficients as positive, and therefore we have written the minus sign inside their definitions explicitly in the equations. We assume that the creation and annihilation operators behave as $c$ numbers, that is, $a_{\alpha}=\sqrt{N_{1}^{\alpha}} e^{i \phi_{1}^{\alpha}}$ where $N_{1}^{\alpha}=\left|a_{\alpha}\right|^{2}$ is the number of particles of species 1 and $\phi_{1}^{\alpha}$ is a phase (similarly $b_{\alpha}=\sqrt{N_{2}^{\alpha}} e^{i \phi_{2}^{\alpha}}$ ). After some algebra, the equations of motion for the number of particles, Eq. (17), and phases, Eq. (18), are obtained from the equations of motion (A13) and (A14). Since we assumed that $m_{1}=m_{2}=m$, and therefore one can use the same localized function for the two components, we obtain that $S=0$ and $\epsilon_{1}=\epsilon_{2}$, and consequently the corresponding terms are absent in Eqs. (17) and (18).

\section{APPENDIX B: EQUATIONS OF MOTION FOR THE IMBALANCE OF POPULATIONS}

Note that Eqs. (17) and (18) can be regarded as obtained from a classical Hamiltonian, and hence $\theta_{k}^{\alpha}$ and $N_{k}^{\alpha}$ are the canonical conjugate variables. Then $\dot{N}_{k}^{\alpha}=\partial H / \partial \theta_{k}^{\alpha}$ and $\dot{\theta}_{k}^{\alpha}=-\partial H / \partial N_{k}^{\alpha}$ with a classical Hamiltonian:

$$
\begin{aligned}
H= & \sum_{k, \alpha}\left\{\left[\frac{1}{2} U_{k} N_{k}^{\alpha} \pm \Gamma+\frac{1}{2} U_{12} N_{3-k}^{\alpha}\right] N_{k}^{\alpha}\right. \\
& \left.-J \sqrt{N_{k}^{\alpha} N_{k}^{\alpha^{\prime}}} \cos \left(\theta_{k}^{\alpha}-\theta_{k}^{\alpha^{\prime}}\right)\right\} \\
& -2 S_{+} \sqrt{N_{2}^{R} N_{1}^{L}} \cos \left(\theta_{1}^{L}-\theta_{2}^{R}\right) \\
& -2 S_{-} \sqrt{N_{2}^{L} N_{1}^{R}} \cos \left(\theta_{1}^{R}-\theta_{2}^{L}\right) \\
& -2 \sum_{\alpha}\left\{\tilde{\delta} \sqrt{N_{1}^{\alpha} N_{2}^{\alpha}} \sin \left(\theta_{1}^{\alpha}-\theta_{2}^{\alpha}\right)\right\},
\end{aligned}
$$

with the upper (lower) sign for $k=1$ (2). Noting that since there is one constant of motion, which is the total number of atoms, we can reduce the number of variables through the following transformation:

$$
\left(\begin{array}{c}
1 \\
z_{1} \\
z_{2} \\
z_{12}
\end{array}\right)=\frac{\mathrm{M}}{N}\left(\begin{array}{c}
N_{1}^{L} \\
N_{1}^{R} \\
N_{2}^{L} \\
N_{2}^{R}
\end{array}\right), \quad\left(\begin{array}{c}
\theta_{N} \\
\theta_{1} \\
\theta_{2} \\
\theta_{12}
\end{array}\right)=-\mathrm{M}\left(\begin{array}{c}
\theta_{1}^{L} \\
\theta_{1}^{R} \\
\theta_{2}^{L} \\
\theta_{2}^{R}
\end{array}\right)
$$

with

$$
M=\left(\begin{array}{rrrr}
1 & 1 & 1 & 1 \\
1 & -1 & 0 & 0 \\
0 & 0 & 1 & -1 \\
1 & 1 & -1 & -1
\end{array}\right)
$$

The set of variables $\left\{z_{i}, \theta_{i}\right\}$ are also canonically conjugate because their Poisson brackets fulfill

$$
\left\{z_{i}, \theta_{j}\right\} \equiv \sum_{k, \alpha}\left(\frac{\partial z_{i}}{\partial N_{k}^{\alpha}} \frac{\partial \theta_{j}}{\partial \theta_{k}^{\alpha}}-\frac{\partial z_{i}}{\partial \theta_{k}^{\alpha}} \frac{\partial \theta_{j}}{\partial N_{k}^{\alpha}}\right)=\delta_{i j} .
$$


From the transformations (B2), one realizes that

$z_{1}=\frac{N_{1}^{L}-N_{1}^{R}}{N}, \quad z_{2}=\frac{N_{2}^{L}-N_{2}^{R}}{N}, \quad z_{12}=\frac{N_{1}-N_{2}}{N}$,

with $N_{k}=N_{k}^{L}+N_{k}^{R}$. The phases associated with $z_{1}, z_{2}$, and $z_{12}$ are, respectively,

$$
\begin{aligned}
& \theta_{1}=\theta_{1}^{R}-\theta_{1}^{L}, \quad \theta_{2}=\theta_{2}^{R}-\theta_{2}^{L}, \\
& \theta_{12}=\theta_{2}^{L}+\theta_{2}^{R}-\left(\theta_{1}^{L}+\theta_{1}^{R}\right) .
\end{aligned}
$$

In the rotated frame [44], $N_{\uparrow}$ and $N_{\downarrow}$, the numbers of bosons in the dressed hyperfine states $\uparrow$ and $\downarrow$, can be written in terms of our quantities as follows:

$$
N_{\sigma}=\frac{1}{2}\left(N_{1}+N_{2} \pm 2 \sum_{\alpha=L, R} \sqrt{N_{1}^{\alpha} N_{2}^{\alpha}} \cos \left(\theta_{1}^{\alpha}-\theta_{2}^{\alpha}\right)\right)
$$

with plus (minus) for $\sigma=\uparrow(\downarrow)$, and $N_{k}^{\alpha}$ and $\theta_{k}^{\alpha}$ solutions of Eqs. (17) and (18). Accordingly, the population imbalance $z_{\uparrow, \downarrow}=\left(N_{\uparrow}-N_{\downarrow}\right) / N$ is related to our variables in the following way:

$$
z_{\uparrow, \downarrow}=\frac{2}{N} \sum_{\alpha=L, R} \sqrt{N_{1}^{\alpha} N_{2}^{\alpha}} \cos \left(\theta_{1}^{\alpha}-\theta_{2}^{\alpha}\right) .
$$

Remarkably, the variables (B4) and (B5) are directly related to the usual Josephson physics. Namely, $z_{k}$ is the population imbalance of component $k$, and $\theta_{k}$ is its corresponding canonical phase. The polarization $z_{12}$ measures the total population transfer between the two components, that is, the population imbalance between the first and the second species. In the absence of spin-orbit coupling the variable $z_{12}$ becomes a constant of motion. Its evolution will thus be intimately related to the effect of the SO term. $\theta_{12}$ is the canonical phase associated with $z_{12}$.

In terms of these variables the Hamiltonian governing the dynamics is $H^{\prime}=2 H / N$, which reads

$$
\begin{aligned}
H^{\prime}= & 2 \Gamma z_{12}-2 J \sum_{k} \sqrt{\left(z_{12} \pm 1\right)^{2}-4 z_{k}^{2}} \cos \left(\theta_{k}\right)+\frac{1}{8} N \sum_{k} U_{k}\left[\left(z_{12} \pm 1\right)^{2}+4 z_{k}^{2}\right]+\frac{1}{4} U_{12} N\left(1+4 z_{1} z_{2}-z_{12}^{2}\right) \\
& +2 S_{+}\left[\left(1-2 z_{2}-z_{12}\right)\left(1+2 z_{1}+z_{12}\right)\right]^{1 / 2} \cos \left[\frac{1}{2}\left(\theta_{1}+\theta_{2}+\theta_{12}\right)\right] \\
& +2 S_{-}\left[\left(1+2 z_{2}-z_{12}\right)\left(1-2 z_{1}+z_{12}\right)\right]^{1 / 2} \cos \left[\frac{1}{2}\left(\theta_{1}+\theta_{2}-\theta_{12}\right)\right] \\
& \mp 2 \bar{\delta} \sum_{k}\left[\left(1 \mp 2 z_{2}-z_{12}\right)\left(1 \mp 2 z_{1}+z_{12}\right)\right]^{1 / 2} \sin \left[\frac{1}{2}\left(\theta_{1}-\theta_{2} \mp \theta_{12}\right)\right]
\end{aligned}
$$

where the first sign that appears holds for $k=1$, and the second for $k=2$. The equations of motion for the atom imbalances (B4) read

$$
\begin{aligned}
\dot{z}_{k}= & -J \sqrt{\left(1 \pm z_{12}\right)^{2}-4 z_{k}^{2}} \sin \left(\theta_{k}\right)-\frac{S_{+}}{2}\left[\left(1-2 z_{2}-z_{12}\right)\left(1+2 z_{1}+z_{12}\right)\right]^{1 / 2} \sin \left[\frac{1}{2}\left(\theta_{1}+\theta_{2}+\theta_{12}\right)\right] \\
& -\frac{S_{-}}{2}\left[\left(1+2 z_{2}-z_{12}\right)\left(1-2 z_{1}+z_{12}\right)\right]^{1 / 2} \sin \left[\frac{1}{2}\left(\theta_{1}+\theta_{2}-\theta_{12}\right)\right] \\
& \mp \frac{1}{2} \bar{\delta}\left[\left(1+2 z_{2}-z_{12}\right)\left(1+2 z_{1}+z_{12}\right)\right]^{1 / 2} \cos \left[\frac{1}{2}\left(\theta_{1}-\theta_{2}+\theta_{12}\right)\right] \\
& \pm \frac{1}{2} \bar{\delta}\left[\left(1-2 z_{2}-z_{12}\right)\left(1-2 z_{1}+z_{12}\right)\right]^{1 / 2} \cos \left[\frac{1}{2}\left(\theta_{1}-\theta_{2}-\theta_{12}\right)\right] \\
\dot{z}_{12}= & -S_{+}\left[\left(1-2 z_{2}-z_{12}\right)\left(1+2 z_{1}+z_{12}\right)\right]^{1 / 2} \sin \left[\frac{1}{2}\left(\theta_{1}+\theta_{2}+\theta_{12}\right)\right]+S_{-}\left[\left(1+2 z_{2}-z_{12}\right)\left(1-2 z_{1}+z_{12}\right)\right]^{1 / 2} \\
& \times \sin \left[\frac{1}{2}\left(\theta_{1}+\theta_{2}-\theta_{12}\right)\right]-\bar{\delta} \sum_{k}\left[\left(1 \pm 2 z_{2}-z_{12}\right)\left(1 \pm 2 z_{1}+z_{12}\right)\right]^{1 / 2} \cos \left[\frac{1}{2}\left(\theta_{1}-\theta_{2} \pm \theta_{12}\right)\right]
\end{aligned}
$$

with the upper sign for $k=1$ and the lower one for $k=2$. The equations for the phases (B5) are

$$
\begin{aligned}
\dot{\theta}_{k}= & N\left(U_{k} z_{k}+U_{12} z_{3-k}\right)+\frac{4 J z_{k} \cos \left(\theta_{k}\right)}{\left[\left(z_{12} \pm 1\right)^{2}-4 z_{k}^{2}\right]^{1 / 2}}+S_{+} \frac{\left[1-2 z_{2}-z_{12}\right]^{ \pm 1 / 2}}{\left[1+2 z_{1}+z_{12}\right]^{ \pm 1 / 2}} \cos \left[\frac{1}{2}\left(\theta_{1}+\theta_{2}+\theta_{12}\right)\right] \\
& +S_{-} \frac{\left[1+2 z_{2}-z_{12}\right]^{ \pm 1 / 2}}{\left[1-2 z_{1}+z_{12}\right]^{ \pm 1 / 2}} \cos \left[\frac{1}{2}\left(\theta_{1}+\theta_{2}-\theta_{12}\right)\right]+\bar{\delta} \frac{\left[1+2 z_{2}-z_{12}\right]^{ \pm 1 / 2}}{\left[1+2 z_{1}+z_{12}\right]^{ \pm 1 / 2}} \sin \left[\frac{1}{2}\left(\theta_{1}-\theta_{2}+\theta_{12}\right)\right] \\
& +\bar{\delta} \frac{\left[1-2 z_{2}-z_{12}\right]^{ \pm 1 / 2}}{\left[1-2 z_{1}+z_{12}\right]^{ \pm 1 / 2}} \sin \left[\frac{1}{2}\left(\theta_{1}-\theta_{2}-\theta_{12}\right)\right]
\end{aligned}
$$




$$
\begin{aligned}
\dot{\theta}_{12}= & 4 \Gamma+N\left[\frac{U_{1}}{2}\left(z_{12}+1\right)+\frac{U_{2}}{2}\left(z_{12}-1\right)-U_{12} z_{12}\right]-2 J \sum_{k} \frac{\left(z_{12} \pm 1\right)}{\sqrt{\left(1 \pm z_{12}\right)^{2}-4 z_{k}^{2}}} \cos \left(\theta_{k}\right) \\
& +2 S_{+} \frac{z_{1}+z_{2}+z_{12}}{\left[1-2 z_{2}-z_{12}\right]^{1 / 2}\left[1+2 z_{1}+z_{12}\right]^{1 / 2}} \cos \left[\frac{1}{2}\left(\theta_{1}+\theta_{2}+\theta_{12}\right)\right] \\
& +2 S_{-} \frac{z_{1}+z_{2}-z_{12}}{\left[1+2 z_{2}-z_{12}\right]^{1 / 2}\left[1-2 z_{1}+z_{12}\right]^{1 / 2}} \cos \left[\frac{1}{2}\left(\theta_{1}+\theta_{2}-\theta_{12}\right)\right] \\
& -2 \bar{\delta} \sum_{k} \frac{z_{1}-z_{2} \pm z_{12}}{\left[1 \pm 2 z_{2}-z_{12}\right]^{1 / 2}\left[1 \pm 2 z_{1}+z_{12}\right]^{1 / 2}} \sin \left[\frac{1}{2}\left(\theta_{1}-\theta_{2} \pm \theta_{12}\right)\right],
\end{aligned}
$$

where, again, the upper (lower) sign corresponds to $k=1(2)$. We have reduced the problem from eight to six equations and checked that these equations give the same numerical results as Eqs. (17) and (18). Notice that if $S_{ \pm}, \Gamma$, and $\bar{\delta}$ vanish, these equations give back those of the two-component two-well problem discussed in Ref. [32]. According to its definition, Eq. (B4), the polarization $z_{12}$ is bounded in the interval $[1,-1]$. The two extremes of this interval correspond to all atoms fully polarized on either internal state 1 or 2 , respectively. For each value of $z_{12}$ it is easy to show that the population imbalance in each component is bounded by $\left|z_{k}\right|=\left(1 \pm z_{12}\right) / 2$, where the minus sign corresponds to $k=2$.

[1] Y. J. Lin, K. Jimenez-Garcia, and I. B. Spielman, Nature (London) 471, 83 (2011).

[2] P. Wang, Z. Q. Yu, Z. Fu, J. Miao, L. Huang, S. Chai, H. Zhai, and J. Zhang, Phys. Rev. Lett. 109, 095301 (2012).

[3] L. W. Cheuk, A. T. Sommer, Z. Hadzibabic, T. Yefsah, W. S. Bakr, and M. W. Zwierlein, Phys. Rev. Lett. 109, 095302 (2012).

[4] Y. A. Bychkov and E. I. Rashba, J. Phys. C 17, 6039 (1984).

[5] G. Dresselhaus, Phys. Rev. 100, 580 (1955).

[6] T. D. Stanescu, B. Anderson, and V. Galitski, Phys. Rev. A 78, 023616 (2008)

[7] G. I. Martone, Y. Li, L. P. Pitaevskii, and S. Stringari, Phys. Rev. A 86, 063621 (2012).

[8] R. Barnett, S. Powell, T. Grass, M. Lewenstein, and S. Das Sarma, Phys. Rev. A 85, 023615 (2012).

[9] V. Galitski and I. B. Spielman, Nature (London) 494, 49 (2013).

[10] Y. Li, G. I. Martone, L. P. Pitaevskii, and S. Stringari, Phys. Rev. Lett. 110, 235302 (2013).

[11] T. Grass, K. Saha, K. Sengupta, and M. Lewenstein, Phys. Rev. A 84, 053632 (2011).

[12] W. S. Cole, S. Zhang, A. Paramekanti, and N. Trivedi, Phys. Rev. Lett. 109, 085302 (2012).

[13] J. Radic, A. Di Ciolo, K. Sun, and V. Galitski, Phys. Rev. Lett. 109, 085303 (2012).

[14] L. Salasnich and B. A. Malomed, Phys. Rev. A 87, 063625 (2013).

[15] V. Achilleos, D. J. Frantzeskakis, P. G. Kevrekidis, and D. E. Pelinovsky, Phys. Rev. Lett. 110, 264101 (2013).

[16] Congjun Wu, Ian Mondragon Shem, and Xiang-Fa Zhou, Chin. Phys. Lett. 28, 097102 (2011).

[17] Xiangfa Zhou, Yi Li, Zi Cai, and Congjun Wu, J. Phys. B 46, 134001 (2013).

[18] M. Burrello and A. Trombettoni, Phys. Rev. Lett. 105, 125304 (2010).

[19] T. Grass, B. Juliá-Díaz, M. Burrello, and M. Lewenstein, J. Phys. B 46, 134006 (2013).

[20] J. P. Vyasanakere, S. Zhang, and V. B. Shenoy, Phys. Rev. B 84, 014512 (2011); M. Gong, S. Tewari, and C. Zhang, Phys. Rev. Lett. 107, 195303 (2011); H. Hu, L. Jiang, X- J. Liu, and H.
Pu, ibid. 107, 195304 (2011); Z.-Q. Yu and H. Zhai, ibid. 107, 195305 (2011); M. Iskin and A. L. Subasi, ibid. 107, 050402 (2011); W. Yi and G.-C. Guo, Phys. Rev. A 84, 031608 (2011); M. Iskin and A. L. Subasi, ibid. 84, 043621 (2011); L. Jiang, X.-J. Liu, H. Hu, and H. Pu, ibid. 84, 063618 (2011); Li Han and C. A. R. Sa de Melo, ibid. 85, 011606(R) (2012).

[21] L. Dell'Anna, G. Mazzarella, and L. Salasnich, Phys. Rev. A 84, 033633 (2011); 86, 053632 (2012).

[22] K. Zhou and Z. Zhang, Phys. Rev. Lett. 108, 025301 (2012).

[23] J. Zhou, W. Zhang, and W. Yi, Phys. Rev. A 84, 063603 (2011); G. Chen, M. Gong, and C. Zhang, ibid. 85, 013601 (2012); X. Yang and S. Wan, ibid. 85, 023633 (2012); L. He and Xu-Guang Huang, Phys. Rev. Lett. 108, 145302 (2012).

[24] A. Barone and G. Paternò, Physics and Applications of the Josephson Effect (Wiley, New York, 1982).

[25] S. Ashhab and C. Lobo, Phys. Rev. A 66, 013609 (2002).

[26] X. Q. Xu, L. H. Lu, and Y. Q. Li, Phys. Rev. A 78, 043609 (2008).

[27] I. I. Satija, R. Balakrishnan, P. Naudus, J. Heward, M. Edwards, and C. W. Clark, Phys. Rev. A 79, 033616 (2009).

[28] B. Juliá-Díaz, M. Guilleumas, M. Lewenstein, A. Polls, and A. Sanpera, Phys. Rev. A 80, 023616 (2009).

[29] G. Mazzarella, M. Moratti, L. Salasnich, M. Salerno, and F. Toigo, J. Phys. B 42, 125301 (2009).

[30] G. Mazzarella, M. Moratti, L. Salasnich, and F. Toigo, J. Phys. B 43, 065303 (2010).

[31] G. Mazzarella, B. A. Malomed, L. Salasnich, M. Salerno, and F. Toigo, J. Phys. B 44, 035301 (2011).

[32] M. Melé-Messeguer, B. Juliá-Díaz, M. Guilleumas, A. Polls, and A. Sanpera, New J. Phys. 13, 033012 (2011).

[33] M. Albiez, R. Gati, J. Fölling, S. Hunsmann, M. Cristiani, and M. K. Oberthaler, Phys. Rev. Lett. 95, 010402 (2005).

[34] R. Gati and M. K. Oberthaler, J. Phys. B 40, R61 (2007).

[35] J. Esteve, C. Gross, A. Weller, S. Giovanazzi, and M. K. Oberthaler, Nature (London) 455, 1216 (2008).

[36] C. Gross, T. Zibold, E. Nicklas, J. Estève, and M. K. Oberthaler, Nature (London) 464, 1165 (2010).

[37] M. F. Riedel, P. Bohi, Y. Li, T. W. Hansch, A. Sinatra, and P. Treutlein, Nature (London) 464, 1170 (2010). 
[38] T. Zibold, E. Nicklas, C. Gross, and M. K. Oberthaler, Phys. Rev. Lett. 105, 204101 (2010).

[39] M. Abbarchi, A. Amo, V. G. Sala, D. D. Solnyshkov, H. Flayac, L. Ferrier, I. Sagnes, E. Galopin, A. Lemaître, G. Malpuech, and J. Bloch, Nat. Phys. 9, 275 (2013).

[40] T. Berrada, S. van Frank, R. Bücker, T. Schumm, J.-F. Schaff, and J. Schmiedmayer, Nat. Commun. 4, 2077 (2013).

[41] A. Smerzi, S. Fantoni, S. Giovanazzi, and S. R. Shenoy, Phys. Rev. Lett. 79, 4950 (1997).

[42] G. J. Milburn, J. Corney, E. M. Wright, and D. F. Walls, Phys. Rev. A 55, 4318 (1997).

[43] D. V. Khomitsky, L. V. Gulyaev, and E. Ya. Sherman, Phys. Rev. B 85, 125312 (2012).

[44] Dan-Wei Zhang, Li-Bin Fu, Z. D. Wang, and Shi-Liang Zhu, Phys. Rev. A 85, 043609 (2012).

[45] M. Albiez, Ph.D. thesis, University of Heidelberg, 2005.
[46] M. Moreno-Cardoner, J. Mur-Petit, M. Guilleumas, A. Polls, A. Sanpera, and M. Lewenstein, Phys. Rev. Lett. 99, 020404 (2007).

[47] H. Qiu, J. Tian, and L. B. Fu, Phys. Rev. A 81, 043613 (2010).

[48] J. Tian, H. Qiu, G. Wang, Y. Chen, and L-B. Fu, Phys. Rev. E 88, 032906 (2013).

[49] B. Juliá-Díaz, M. Mele-Messeguer, M. Guilleumas, and A. Polls, Phys. Rev. A 80, 043622 (2009).

[50] J. Gillet, M. A. Garcia-March, Th. Busch, and F. Sols, Phys. Rev. A 89, 023614 (2014).

[51] R. Citro and A. Naddeo, arXiv:1405.5356.

[52] R. W. Spekkens and J. E. Sipe, Phys. Rev. A 59, 3868 (1999).

[53] M. A. Garcia-March, D. R. Dounas-Frazer, and L. D. Carr, Phys. Rev. A 83, 043612 (2011).

[54] M. A. Garcia-March, D. R. Dounas-Frazer, and L. D. Carr, Front. Phys. 7, 131 (2012). 